\title{
Integrated Flight/Structural Mode Control for Very Flexible Aircraft Using L1 Adaptive Output Feedback Controller
}

\author{
Jiaxing Che ${ }^{*}$, \\ University of Connecticut, Storrs, CT 06269 \\ Irene M. Gregory ${ }^{\dagger}$ \\ NASA Langley Research Center, Hampton, VA 23681 \\ Chengyu Cao * \\ University of Connecticut, Storrs, CT 06269
}

\begin{abstract}
This paper explores application of adaptive control architecture to a light, high-aspect ratio, flexible aircraft configuration that exhibits strong rigid body/flexible mode coupling. Specifically, an $L_{1}$ adaptive output feedback controller is developed for a semi-span wind tunnel model capable of motion. The wind tunnel mount allows the semi-span model to translate vertically and pitch at the wing root, resulting in better simulation of an aircraft's rigid body motion. The control objective is to design a pitch control with altitude hold while suppressing body freedom flutter. The controller is an output feedback nominal controller (LQG) augmented by an $L_{1}$ adaptive loop. A modification to the $L_{1}$ output feedback is proposed to make it more suitable for flexible structures. The new control law relaxes the required bounds on the unmatched uncertainty and allows dependence on the state as well as time, i.e. a more general unmatched nonlinearity. The paper presents controller development and simulated performance responses. Simulation is conducted by using full state flexible wing models derived from test data at 10 different dynamic pressure conditions. An $L_{1}$ adaptive output feedback controller is designed for a single test point and is then applied to all the test cases. The simulation results show that the $L_{1}$ augmented controller can stabilize and meet the performance requirements for all 10 test conditions ranging from 30 psf to 130 psf dynamic pressure.
\end{abstract}

\section{Introduction}

he next generation of efficient subsonic aircraft will need to be both high aspect ratio and light weight, and the associated structural flexibility presents both challenges and opportunities. An example of advanced vehicles under consideration is illustrated in Fig. $1^{1}$. Robust active aeroelastic control and Gust Load Alleviation (GLA) have a potential to substantially reduce weight by relaxing structural strength requirements on the wings. However, current tools such as traditional linear aeroelastic and aeroservoelastic analysis methods are inadequate to reliably predict aeroelastic stability and assess active aeroelastic control effectiveness in this type of vehicle. The type of vehicle represents a nonlinear stability and control problem involving complex interactions among the flexible structure, unsteady aerodynamics, flight control system, propulsion system, the environmental conditions, and vehicle flight dynamics. Furthermore, because of the inherent flexibility of the aircraft, the lower order structural mode frequencies are of the same order as the rigid-body mode frequencies. The close proximity of flexible and rigid-body

\footnotetext{
${ }^{*}$ Ph.D Student, Department of Mechanical Engineering, University of Connecticut. AIAA member. Jiaxing.Che@engineer.uconn.edu

${ }^{\dagger}$ Senior Research Engineer, Dynamic Systems and Control Branch, MS 308, NASA Langley Research Center, Irene.M.Gregory@nasa.gov, AIAA Assoc. Fellow.

ॠ Assistant Professor, Department of Mechanical Engineering, University of Connecticut. Ccao@engr.uconn.edu 
dynamics does not allow for the more traditional designs based on the separation between these two set of dynamics. Hence, this trend in aeronautics and high level of uncertainty associated with available aircraft design tools present an important challenge for adaptive control theory. The fundamental theory of $L_{1}$ adaptive control is introduced in Ref [2-7]. State feedback $L_{1}$ adaptive control has been used in a number of challenging applications, the theory verified and design process matured against real world challenges. For example, the $L_{1}$ adaptive control has been recently used to enable real-time dynamic modeling of the edges of a flight envelope of a dynamically scaled model of a generic transport aircraft with conventional configuration ${ }^{8}$. On the other hand, the output feedback $L_{1}$ adaptive control is in the early stages of application to such new challenges as very flexible aircraft.

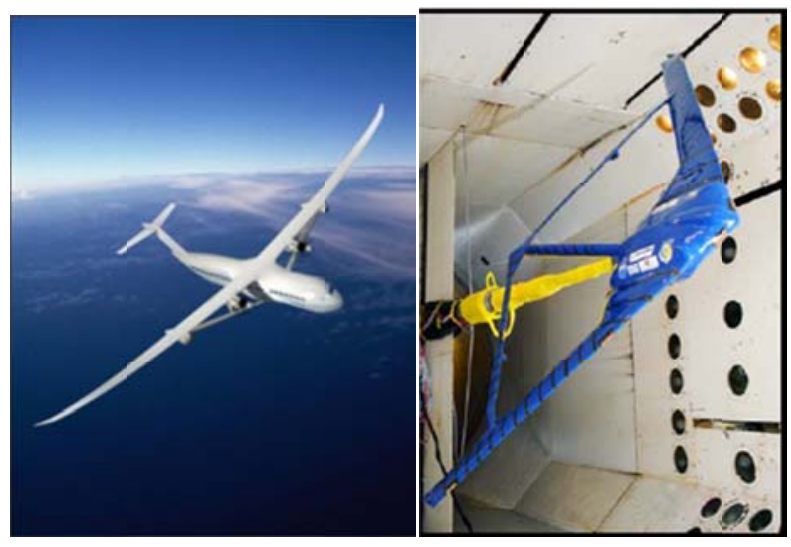

Figure 1. New generation of efficient subsonic aircraft.

This paper presents an application of a recent extension of the $L_{1}$ adaptive output feedback controller in Ref [9] to an advanced highly flexible aircraft configuration. The extension of $L_{1}$ output feedback control design is to a class of nonlinear systems in the presence of unknown state-dependent and time-varying nonlinearities. Moreover, the controller considers bounded time-varying unmatched uncertainty. The control algorithm consists of an output predictor which is designed to predict the system's output with arbitrary accuracy and a control law which is used to control the predictor output instead of the actual system's output. It is shown that the adaptive output feedback controller ensures uniformly bounded output tracking for the system. The difference between the output predictor and the actual system's output is bounded, and this performance bound can be made arbitrarily small by reducing the step size of integration. However, this particular extension is not the most suitable formulation to deal with highly flexible vehicles. The control law is based on dynamic inversion, which will cancel the effects of uncertainties only on the output. In output feedback, the effect on the state cannot be ensured, and in fact, the feedback can destabilize the entire system. It is for this reason that the unmatched uncertainty is a bounded function of time. In this paper, further modification to the $L_{1}$ output feedback is proposed, in particular changing the control law itself. The new control law will relax the required bounds on the unmatched uncertainty and will allow dependence on the state as well as time, i.e., a more general unmatched nonlinearity. This formulation, with the usual assumptions on observability and controllability, will make the new $L_{1}$ output feedback more suitable for dealing with highly flexible vehicles. Control law design details are discussed in section IV. To demonstrate the potential of the proposed $L_{1}$ output feedback approach, a semi-span high aspect ratio flexible aircraft wind tunnel model from Ref. [10] is revisited. Numerical simulation results for a very flexible aircraft configuration illustrate the algorithm's performance as well as quantify the achievable performance bound as a function of the control computer CPU for this class of vehicles.

\section{Model Description}

The dearth of publicly available control-centric nonlinear models for very flexible aircraft led the authors to revisit the model from Ref. [10]. The details of the physical wind tunnel model can be found in Ref. [10]. The range of vertical motion is constrained to \pm 12 inches, hard stop to hard stop, and the model angle of attack is limited by loading considerations to single digits in degrees. The wind tunnel model is instrumented with accelerometers along 
the spar, strain gauges at the root and mid-spar, a rate gyro at the wing tip, and a rate gyro and accelerometers at the tunnel attachment point.

The mathematical model is linear and includes rigid body translational and rotational displacements and velocities $(z, \theta, w, q)$, as well as twelve flexible modes. The flexible modes are represented by generalized displacements, $\eta_{i}$, and velocities, $\dot{\eta}_{i}$. There were 10 test points for the flexible vehicle wind tunnel model, each corresponding to a different dynamic pressure ranging from 30 psf to 130 psf shown in Table 1 , and an associated linear model described above. The simulation consists of linear models with third order actuator dynamics, typical of aeroservoelastic models, included for each of the control surfaces in the simulation.

Table 1. Dynamic pressure for different test points.

\begin{tabular}{|c|c|c|c|c|c|c|c|c|c|c|}
\hline $\begin{array}{c}\text { Test Point } \\
\text { Index }\end{array}$ & 1 & 2 & 3 & 4 & 5 & 6 & 7 & 8 & 9 & 10 \\
\hline $\begin{array}{c}\text { Dynamic } \\
\text { Pressure (psf) }\end{array}$ & 30 & 35 & 40 & 45 & 50 & 60 & 70 & 80 & 90 & 130 \\
\hline
\end{tabular}

The general structure of the flexible model is given by

$$
\left[\begin{array}{c}
\dot{x}_{r} \\
\dot{x}_{e} \\
\dot{x}_{\text {lag }} \\
\dot{x}_{\delta}
\end{array}\right]=\left[\begin{array}{cccc}
A_{r} & A_{r}^{e} & A_{r}^{\text {lag }} & A_{r}^{\delta} \\
A_{e}^{r} & A_{e} & A_{e}^{\text {lag }} & A_{e}^{\delta} \\
A_{\text {lag }}^{r} & A_{\text {lag }}^{e} & A_{\text {lag }} & A_{\text {lag }}^{\delta} \\
0 & 0 & 0 & A_{\delta}
\end{array}\right]\left[\begin{array}{c}
x_{r} \\
x_{e} \\
x_{\text {lag }} \\
x_{\delta}
\end{array}\right]+\left[\begin{array}{c}
0 \\
0 \\
0 \\
B_{c m d}
\end{array}\right] \delta_{c m d}
$$

where $x_{r}$ represents rigid body position and rates, $x_{e}$ represents elastic mode deflections and rates, $x_{\text {lag }}$ represents aerodynamic lag states, and $x_{\delta}$ represents actuator states. For control design purposes, the model is residualized to eliminate lag states and then is further reduced by eliminating higher frequency flexible modes. Furthermore, the actuator dynamics are neglected, and as a result, the control design model is reduced from 112 to 12 state variables consisting of 2 rigid (half-span) modes and 4 flexible modes. Thus, the model used for design has the format

$$
\begin{aligned}
& {\left[\begin{array}{c}
\dot{\bar{X}}_{r} \\
\dot{\bar{X}}_{e}
\end{array}\right]=\left[\begin{array}{cc}
A_{r} & A_{r}^{e} \\
A_{e}^{r} & A_{e}
\end{array}\right]\left[\begin{array}{l}
\bar{X}_{r} \\
\bar{X}_{e}
\end{array}\right]+\left[\begin{array}{c}
A_{\delta}^{r} \\
A_{\delta}^{e}
\end{array}\right] \delta} \\
& \bar{y}=\left[\begin{array}{ll}
C_{r} & C_{e}
\end{array}\right]\left[\begin{array}{l}
\bar{X}_{r} \\
\bar{X}_{e}
\end{array}\right]+D \delta
\end{aligned}
$$

where $\delta=\left[\delta_{L E}, \delta_{T E 1}, \delta_{T E 2}, \delta_{T E 3}, \delta_{T E 4}\right]^{T}$ and $y$ is the output of sensors described in Ref [10]. In order to improve tracking and add damping into the system, a proportional plus integral (PI) control structure that tracks vertical displacement is chosen for the baseline controller. In order to create a model structure compatible with $L_{1}$ output feedback several of the sensor measurements, combined in such a way as to isolate flexible wing modes, are added to the system in Eq. (2) as states. In addition, the integrator on position is augmented to the system in Eq. (2) resulting in a new system structure given by

$$
\begin{aligned}
& \dot{x}=A_{m} x+B_{m} u \\
& y=C_{m} x
\end{aligned}
$$

where $u=\delta$ is a vector of control inputs (one leading edge and four trailing edges), $y$ is a vector of sensor and integrator outputs, and $A_{m} \in R^{16 \times 16}, B_{m} \in R^{16 \times 5}, C_{m} \in R^{8 \times 16}$ are matrices with appropriate dimensions. 


\section{Control Objective and Design Approach}

Similar to Ref [10], the general control approach is to design a robust linear controller at one of the test points and then use adaptive control as an augmentation on the nominal control system. In this approach, the role of adaptation is to maintain the desired system performance defined by the nominal controller/vehicle closed-loop dynamics, in the presence of uncertainties or unknown variation in plant dynamics. The objective is to control the first and second bending modes of the wing, pictured in Fig. 2, while executing vertical position hold and controlling pitch moment at the pivot point.

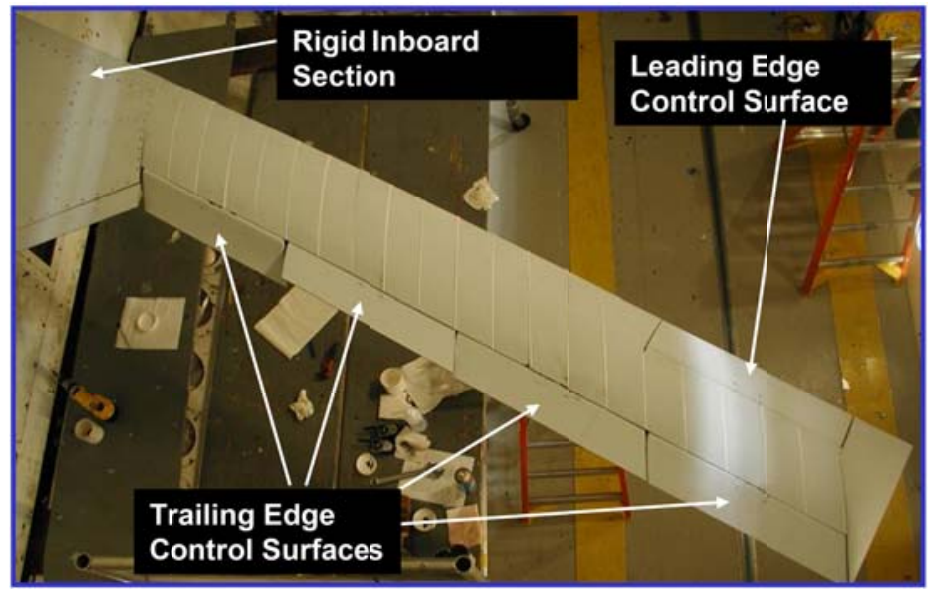

Figure 2. Wind tunnel model

An overview of the control architecture is illustrated in Fig. 3. The $L_{1}$ adaptive control augmentation uses a fast adaption scheme to estimate the unmodeled uncertainties. The control law for disturbance rejection uses a low-pass filter mechanism to get a band-limited augmentation control signal. As an augmentation controller, LQG and $L_{1}$ share the same state predictor (this will be addressed in more detail in the next section). When the plant model is close to the model used for the LQG controller design, the output of the $L_{1}$ adaptive law is almost zero. The $L_{1}$ adaptive controller accounts for all the model uncertainty and unmolded dynamics with an adaptive parameter. From a high level perspective, the LQG is controlling the exact plant as the one used for the LQG parameter design, and the $L_{1}$ adaptive controller is dealing with the model mismatch and unmodeled dynamics. The low-pass filter is tuned in a tradeoff between performance and stability.

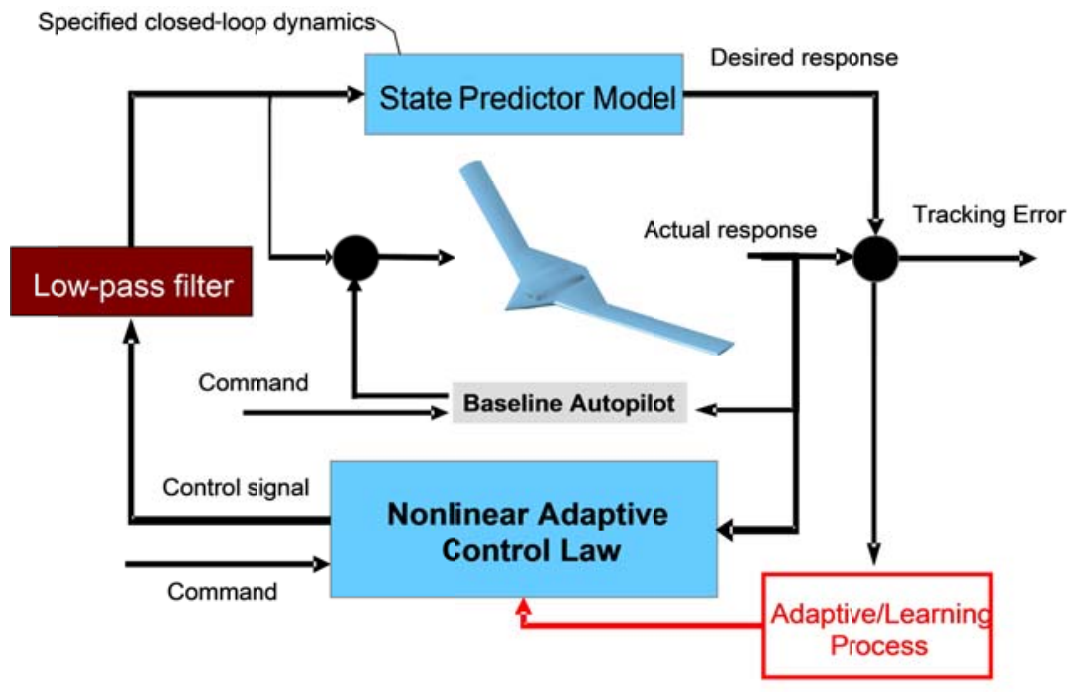

Figure 3. $L_{1}$ adaptive control architecture for stability augmentation. 


\section{IV. $\quad L_{1}$ Augmentation Adaptive Controller Design}

Consider the following system dynamics

$$
\begin{aligned}
& \dot{x}(t)=A x(t)+\theta_{z} z(t)+\theta_{w} w_{g}(t)+B u(t) \\
& \dot{z}(t)=f(x(t), z(t), t) \\
& y(t)=C x(t)
\end{aligned}
$$

where $x(t) \in R^{n}$ are system states, $y \in R^{l}$ are output variables available for feedback, $u(t) \in R^{p}$ are control signals, $z(t) \in R^{m}$ are bounded input bounded output (BIBO) unmodeled system dynamics, $w_{g}(t)$ is a bounded external disturbance, $(A, B, C)$ are unknown matrices of appropriate dimensions, $A, \theta_{z}, \theta_{w}$ represent unknown parameters with given conservative bounds, and $f$ is a Lipchitz continuous unknown function.

The control objective is to design an augmentation adaptive output feedback controller, using only available measurements, $y(t)$, such that $y(t)$ tracks a given bounded reference input $r(t)$ with desired transient and asymptotic performance.

Note that given a Hurwitz matrix $A_{m}$ such that the triple $\left(A_{m}, B_{m}, C_{m}\right)$ is controllable and observable, Eq. (4) can be rewritten as

$$
\begin{aligned}
\dot{x}(t) & =A_{m} x(t)+B_{m} u(t)+\sigma(t) \\
\dot{z}(t) & =f(x(t), z(t), t) \\
y(t) & =C_{m} x(t)
\end{aligned}
$$

where $\sigma(t)$ is a vector of time varying signals that includes all the uncertainties and the disturbances, i.e

$$
\sigma(t)=\left(A-A_{m}\right) x(t)+\theta_{z} z(t)+\theta_{w} w_{g}(t)+\left(B-B_{m}\right) u(t)
$$

The design process is to use the parameters $\left(A_{m}, B_{m}, C_{m}\right)$, from Eq. (3), to design an LQG baseline controller to achieve the desired tracking performance in the nominal system. Then an $L_{1}$ output feedback controller is designed to deal with the model uncertainties and the disturbances in the $\sigma(t)$ term.

\section{A. LQG Baseline Controller Design:}

The LQG controller is the combination of a Kalman filter with a linear-quadratic regulator (LQR). The detailed design procedure for an LQG is omitted here, but an overview using the above problem formulation is provided below. The Kalman filter is

$$
\begin{aligned}
& \dot{\hat{x}}(t)=A_{m} \hat{x}(t)+B_{m} u(t)+L(t)\left(y-C_{m} \hat{x}(t)\right) \\
& \hat{y}=C_{m} \hat{x}(t)
\end{aligned}
$$

where $\hat{x}(t)$ is the estimated state and $L(t)$ is the observer gain for the Kalman filter. Tuning the covariance matrix of process noise and measurement noise in the LQG problem formulation can change the performance of the Kalman filter.

The LQR controller design uses the estimated state, $\hat{x}(t)$. The baseline control law is defined as

$$
u=K \hat{x}(t)
$$

The dynamic performance and control effort is tuned by choosing different performance and control effort matrices $\mathrm{Q}$ and R. The overall baseline control law is defined in Eq. (8) with the estimated state, $\hat{x}(t)$, generated by Eq. (7). There are several limitations to the LQG design. The LQG design is optimal when using the real plant model, $(A, B, C)=\left(A_{m}, B_{m}, C_{m}\right)$. In reality $(A, B, C)$ are unknown. The LQR controller has limited stability when $(A, B, C)$ 
have large deviations from $\left(A_{m}, B_{m}, C_{m}\right)$. The white process noise assumption on the Kalman filter is also not satisfied in this system. Hence, the LQG design has a limited performance and stability margin due to the invalid white noise assumption.

\section{B. $L_{1}$ Control Augmentation Design:}

\section{State Predictor:}

In addition to Eq. (7), a vector of adaptive parameters, $\hat{\sigma}$, is introduced to the predictor.

$$
\begin{aligned}
& \dot{\hat{x}}(t)=A_{m} \hat{x}(t)+B_{m} u(t)+L(t)\left(y-C_{m} \hat{x}(t)\right)+\hat{\sigma} \\
& \hat{y}=C_{m} \hat{x}(t)
\end{aligned}
$$

\section{Adaptive Law:}

The adaptive law updates $\hat{\sigma}$ as piece-wise constant signal such that output tracking, $\hat{y}(t) \approx y(t)$ is ensured. Prior to introducing the adaptive law, certain variables need to be defined as follows.

Since $A_{m}$ is Hurwitz, there exists $P=P^{\top}>0$ that satisfies the algebraic Lyapunov equation

$$
\begin{aligned}
& A_{m}^{\top} P+P A_{m}=-Q \\
& Q>0
\end{aligned}
$$

From the properties of $P$, it follows that there exists a non-singular $\sqrt{P}$ such that

$$
P=(\sqrt{P})^{\top} \sqrt{P}
$$

Given $C_{m}^{\top}(\sqrt{P})^{-1}$, let $D$ be an $(n-1) \times n$ matrix that contains the null space of $C_{m}^{\top}(\sqrt{P})^{-1}$ such that $D\left(C_{m}^{\top}(\sqrt{P})^{-1}\right)^{\top}=0$, and further let $\Lambda=\left[\begin{array}{c}C_{m}^{\top} \\ D \sqrt{P}\end{array}\right]$.

Let $T$ be any positive constant, which can be associated with the sampling rate of the available CPU, and let $\mathbf{1}_{1}=\left[\begin{array}{ll}I_{m \times m} & O_{m \times(n-m)}\end{array}\right]^{T} \in \mathrm{R}^{n \times m}$ be the matrix with first sub-matrix an identity matrix and all other elements zero $\left(\mathbf{1}_{1}\right.$ matrices are designed to match the dimensions in Eq. (13) ). Let $\tilde{y}(t)=\hat{y}(t)-y(t)$ be output tracking error, then the update law for $\hat{\sigma}(t)$ is given by

$$
\begin{aligned}
& \hat{\sigma}(t)=\hat{\sigma}(i T), \quad t \in[i T,(i+1) T) \\
& \hat{\sigma}(i T)=-\Phi^{-1}(T) \mu(i T), \\
& i=0,1,2, \cdots
\end{aligned}
$$

where $\Phi(T)$ and $\mu(i T)$ are defined as

$$
\Phi(T)=\int_{0}^{T} \mathrm{e}^{\Lambda A_{m} \Lambda^{-1}(T-\tau)} \Lambda d \tau, \quad \mu(i T)=\mathrm{e}^{\Lambda A_{m} \Lambda^{-1} T} \mathbf{1}_{1} \tilde{y}(i T), i=0,1,2,3, \cdots
$$

Letting $B_{u m} \in R^{n \times(n-m)}$ be the null space of $B_{m} \in R^{n \times m}$, so that $B_{m}^{T} B_{u m}^{T}=0$ and $\operatorname{rank}\left(\left[\begin{array}{ll}B_{m} & B_{u m}\end{array}\right]\right)=\mathrm{n}$, the adaptive parameter $\hat{\sigma}(t)$ can be further decoupled into matched and unmatched terms

$$
\left[\begin{array}{c}
\hat{\sigma}_{m}(i T) \\
\hat{\sigma}_{u m}(i T)
\end{array}\right]=\left[\begin{array}{ll}
B_{m} & B_{u m}
\end{array}\right]^{-1} \hat{\sigma}(i T)
$$


In the following control law design, the matched disturbance, $\hat{\sigma}_{m}(i T)$, and unmatched disturbance, $\hat{\sigma}_{u m}(i T)$, are filtered with different filter bandwidths. This approach defining and updating compensation for matched and unmatched uncertainty in the state feedback formulation has been successfully flight tested on NASA GTM aircraft, e.g. see $\operatorname{Ref}[11]$.

\section{Control law design:}

In addition to the baseline LQG control signal, $u_{b}$, in Eq. (8), there are two augmentation control signals, $u_{m}(t)$ and $u_{u m}(t)$, for matched and unmatched uncertainties respectively. This control law is inspired by a successful application in Ref [11]. In this paper the control law is further modified to be suitable for highly flexible vehicles.

The overall control law is generated as

$$
\begin{aligned}
& u(t)=u_{m}(t)+u_{u m}(t)+u_{b}(t) \\
& u_{m}(s)=-C_{1}(s) \hat{\sigma}_{m}(s) \\
& u_{u m}(s)=-C_{2}(s) W \hat{\sigma}_{u m}(s)
\end{aligned}
$$

where $H_{1}(s)=C\left(s I-A_{m}\right)^{-1} B_{m}, H_{2}(s)=C\left(s I-A_{m}\right)^{-1} B_{u m}$, and $\mathrm{W}$ is the DC gain matrix of the system $H_{1}^{-1}(s) H_{2}(s) . C_{1}(s), C_{2}(s)$ are the square low pass filter matrix with unity gain for the diagonal element. $C_{1}(s)$ is the filter matrix for the matched channel and $C_{2}(s)$ is the filter matrix for unmatched channel.

The design objective of $u_{m}(t)$ and $u_{u m}(t)$ is to compensate for the effect of model mismatch. This decomposition enables filter design of different bandwidths for the matched channel and unmatched channel separately. Compensating the unmatched uncertainties usually leads to stability challenges. By tuning these two filter bandwidths of $C_{1}(s), C_{2}(s)$ separately, the stability margin of the controller can be configured to match the actual range of the system parameter. A detailed analysis is summarized in Ref [12].

An important modification in this paper is that instead of dynamic inversion of $H_{1}^{-1}(s) H_{2}(s)$, the DC gain matrix, W, of the system $H_{1}^{-1}(s) H_{2}(s)$ is inverted instead. This modification is necessary because the flexible vehicle model structure has a high system dimension and high frequency lightly damped modes. Under nominal conditions, dynamic inversion will improve the tracking performance, but the control signal will easily excite the high frequency mode shapes, especially when dealing with high system dimensions with large parameter deviations due to wide range of encompassed flight conditions. The frequencies of the flexible modes are uncertain and the first and second flexible modes have frequencies close to rigid body dynamics and are close to each other making cancellation by inversion completely impractical. In addition, the rank of the controllability matrix is low compared to the real system dimensions, which means many of the high frequency mode shapes are nearly uncontrollable. To avoid exciting the uncertain flexible mode frequencies, the dynamic inversion is changed to a static inversion, thus, only compensating the static error which makes more sense for the flexible structure. This results in a different stability condition for the $L_{1}$ output feedback controller.

Revisiting the baseline control signal $u_{b}(s)$ in terms of the system formulation in Eq. (9) is given by

$$
u_{b}(s)=K \hat{x}(s)+K_{g} K_{p}\left(C_{c}(s) r(s)-y(s)\right)
$$

where $\mathrm{K}$ is the feedback gain from the LQR controller design, $\hat{x}(t)$ is the estimated state from Eq. (6), and $\mathrm{K}_{g}=-\left(C A_{m}^{-1} B\right)^{-1}$. This choice for $\mathrm{K}_{g}$ ensures that the diagonal elements of the transfer matrix $\left[C\left(s I-A_{m}\right)^{-1} B_{m} K_{g}\right]$ have DC gain equal to one, while the off-diagonal elements have zero DC gain. $K_{p}$ is a proportional design factor used to add damping to the adaptation loop inside the controller and to tune frequency response and robustness margins when there is model parameter deviation. $C_{c}(s)$ is a command filter with unity DC gain. Together with the $K_{p}$ design, the baseline control law, $u_{b}(t)$, makes the system behavior close to the command filter $C_{c}(s) . r(s)$ is the reference input signal. 


\section{Simulation Results}

The controller was evaluated in simulation on the original full state linear wind tunnel models derived at 10 different test dynamic pressures. The simulation also includes third order actuator dynamics and time-delay in the sensor feedback loop. The simulation time step and, hence, the sampling time was set at $T=0.005$. For the purposes of this publication, the magnitude of control deflections and vehicle attitude has been normalized with respect to the trim values.

Let $\left(A_{i r}, B_{i r}, C_{i r}\right), i r=1, \ldots .10$, where $A \in R^{112 \times 112}, B \in R^{112 \times 5}, C \in R^{8 \times 12}$ represent the full state linear model derived from the wind tunnel test at a given test dynamic pressure with ir associated with a particular dynamic pressure from Table 1. Let $\left(A_{m i}, B_{m i}, C_{m i}\right), i=1,2, \ldots 10$ represent the reduced order model used for controller design at a particular test dynamic pressure with $i$ associated with a particular dynamic pressure from Table 1.

An LQG controller is designed as the baseline controller for each test point. The particular controller is then evaluated at different test conditions in addition to the nominal test condition. In other words, a particular baseline control signal $u_{b}(t)$, given in Eq. (18), is designed using $\left(A_{m i}, B_{m i}, C_{m i}\right), i=10$ is tested for the plant $\left(A_{i r}, B_{i r}, C_{i r}\right), i r=1, \ldots . .10$. The stability results for each LQG baseline controller are given in Table 2 where shaded cells indicate matched design and evaluation conditions, e.g. LQG controller designed using reduced order model at $130 \mathrm{psf}$ is evaluated on full order linear model at $130 \mathrm{psf}(i \mathrm{r}=10)$. As seen from Table 2, in the simulation the baseline controller is limited in its ability to stabilize plant models different from the model used to design the controller. For example, the LQG controller designed for model 10 (130 psf test condition) can stabilize full linear models at 90 and 80 psf $(i r=9,8)$ as indicated by checkmarks in Table 2, but cannot stabilize models for dynamic pressure below $80 \mathrm{psf}$ (ir=7-1, 70 to $30 \mathrm{psf}$ test conditions). The LQG controller does somewhat better in the middle ranges of tested dynamic pressure by stabilizing the system up to 3 test points below its design condition covering the range of 90 to $60 \mathrm{psf}$. As dynamic pressure drops towards minimum necessary for flight, the range over which LQG can provide stability also decreases. It is interesting to note that in all case, the baseline LQG stabilizes the vehicle for dynamic pressure at or below its design condition. There are two reasons for the unstable response. One is the large parameter variation with dynamic pressure especially in the control effectiveness. The other challenge is the unmodeled dynamics not captured in the reduced order model used for control law design. In summary, the baseline controller designed with reduced order model $i$ appears to stabilize the system for ir $=i$,ir $=i-1, i r=i-2$, and in some cases for $i r=i-3$, which means when the real plant changes, the baseline controller can work for at most 4 test conditions.

Table 2. Stability of baseline LQG controller designed for test conditions.

\begin{tabular}{|c|c|c|c|c|c|c|c|c|c|c|c|}
\hline & \multicolumn{10}{|c|}{ Index number of model used for the full state plant (used for evaluation) } \\
\hline & & 1 & 2 & 3 & 4 & 5 & 6 & 7 & 8 & 9 & 10 \\
\hline \multirow{10}{*}{ 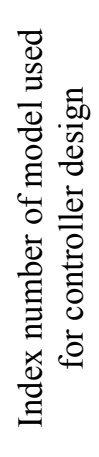 } & 1 & $\sqrt{ }$ & & & & & & & & & \\
\hline & 2 & $\sqrt{ }$ & $\sqrt{ }$ & & & & & & & & \\
\hline & 3 & $\sqrt{ }$ & $\sqrt{ }$ & $\sqrt{ }$ & & & & & & & \\
\hline & 4 & $\sqrt{ }$ & $\sqrt{ }$ & $\sqrt{ }$ & $\sqrt{ }$ & & & & & & \\
\hline & 5 & & $\sqrt{ }$ & $\sqrt{ }$ & $\sqrt{ }$ & $\sqrt{ }$ & & & & & \\
\hline & 6 & & & $\sqrt{ }$ & $\sqrt{ }$ & $\sqrt{ }$ & $\sqrt{ }$ & & & & \\
\hline & 7 & & & & $\sqrt{ }$ & $\sqrt{ }$ & $\sqrt{ }$ & $\sqrt{ }$ & & & \\
\hline & 8 & & & & & $\sqrt{ }$ & $\sqrt{ }$ & $\sqrt{ }$ & $\sqrt{ }$ & & \\
\hline & 9 & & & & & & $\sqrt{ }$ & $\sqrt{ }$ & $\sqrt{ }$ & $\sqrt{ }$ & \\
\hline & 10 & & & & & & & & $\sqrt{ }$ & $\sqrt{ }$ & $\sqrt{ }$ \\
\hline
\end{tabular}

In Eq. (18) $r \in R$ is the command for vertical position in the wind tunnel. Figs. 4 to 10 illustrate the response of the baseline controller designed with $\left(A_{m i}, B_{m i}, C_{m i}\right), i=10(130 \mathrm{psf})$ when the real plant varies from 130 psf to 50 psf, corresponding to $i r=10$ to $i r=5$. The discussion is similar to the example describing the results in Table 2 . When ir $=10=i$, which means the full state model $\left(A_{i r}, B_{i r}, C_{i r}\right)$ is at the same test condition as the design and is close to the model $\left(A_{m i}, B_{m i}, C_{m i}\right)$ used for controller design, the system response in Fig. 4 tracks with no error. When the real plant is changed to ir $=9,8$, there are some higher frequency oscillations that become apparent in the 
pitch rate and in control signal, especially the leading edge (LE) as shown in Figs. 5 and 6 . When $i r=7$, the baseline controller can no longer stabilize the system. Fig. 7 illustrates that as ir gets further from the design condition $i$, the closed loop system goes unstable much more rapidly.

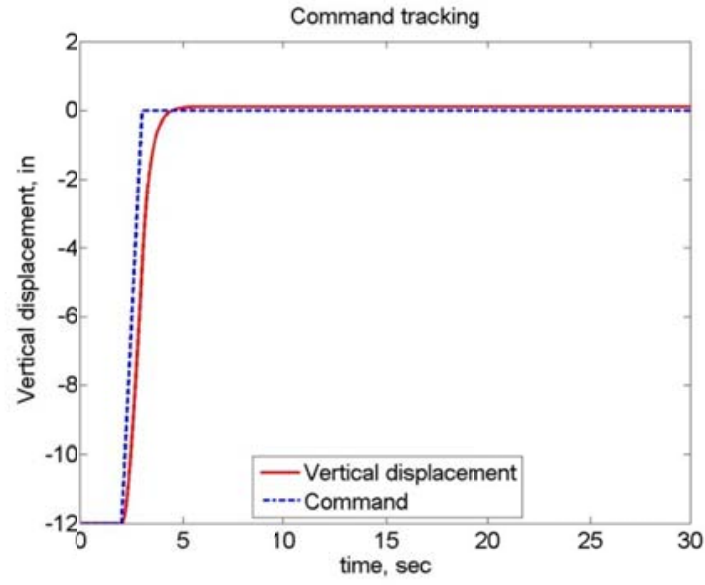

(a) Vertical tunnel position (in)

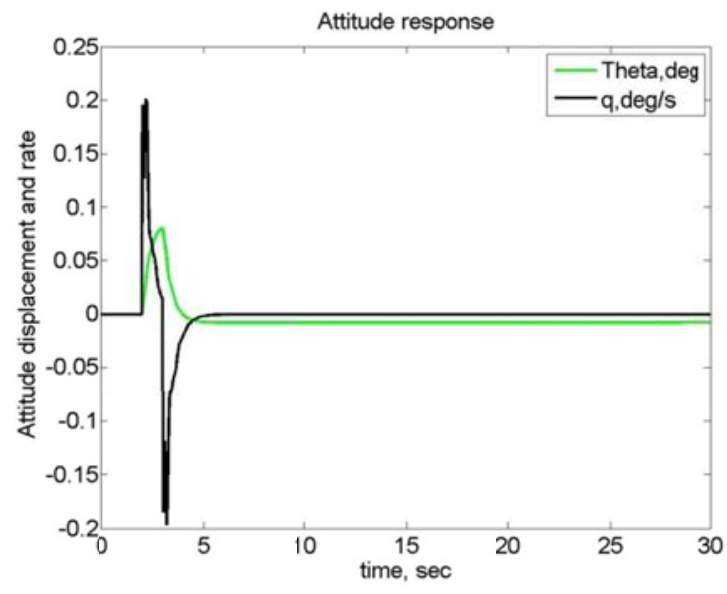

(c) Model attitude $\theta(\mathrm{deg})$ and $\mathrm{q}(\mathrm{deg} / \mathrm{s})$

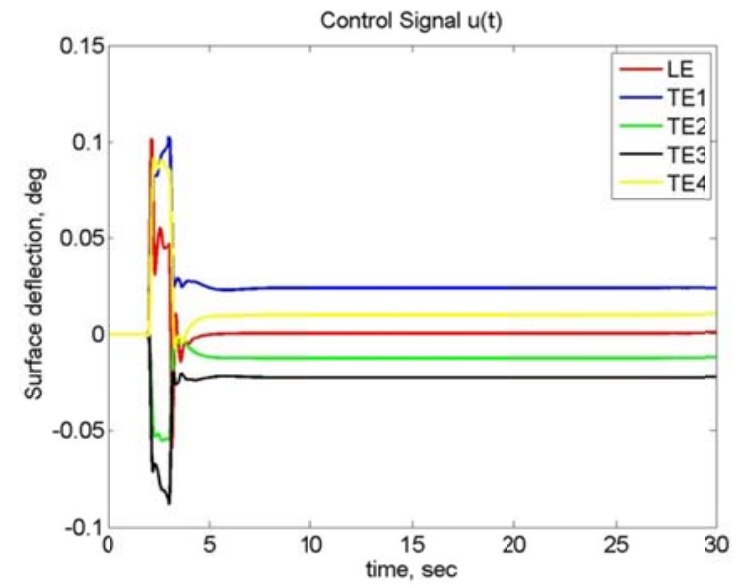

(b) Control deflections (deg)

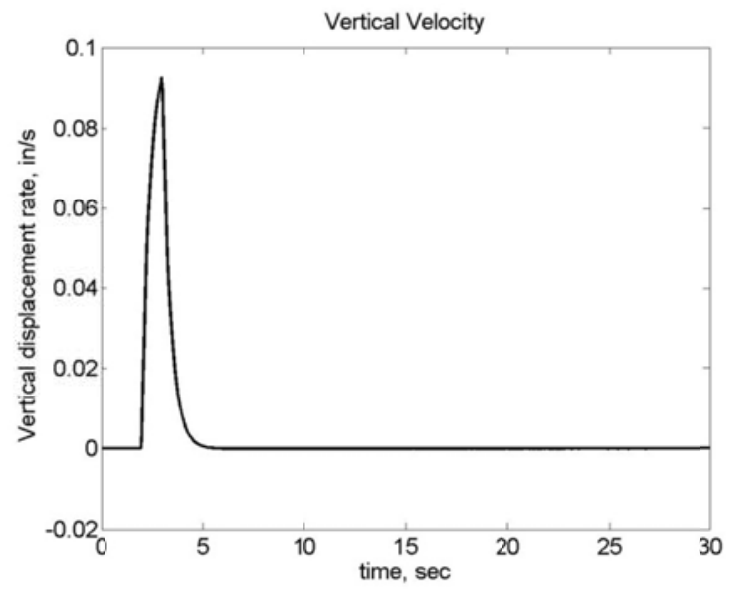

(d) Vertical velocity $\mathrm{Vz}(\mathrm{in} / \mathrm{s})$

Figure 4. Measured output responses for the baseline controller (130psf) evaluated at $130 \mathrm{psf}(\mathrm{ir}=10)$.
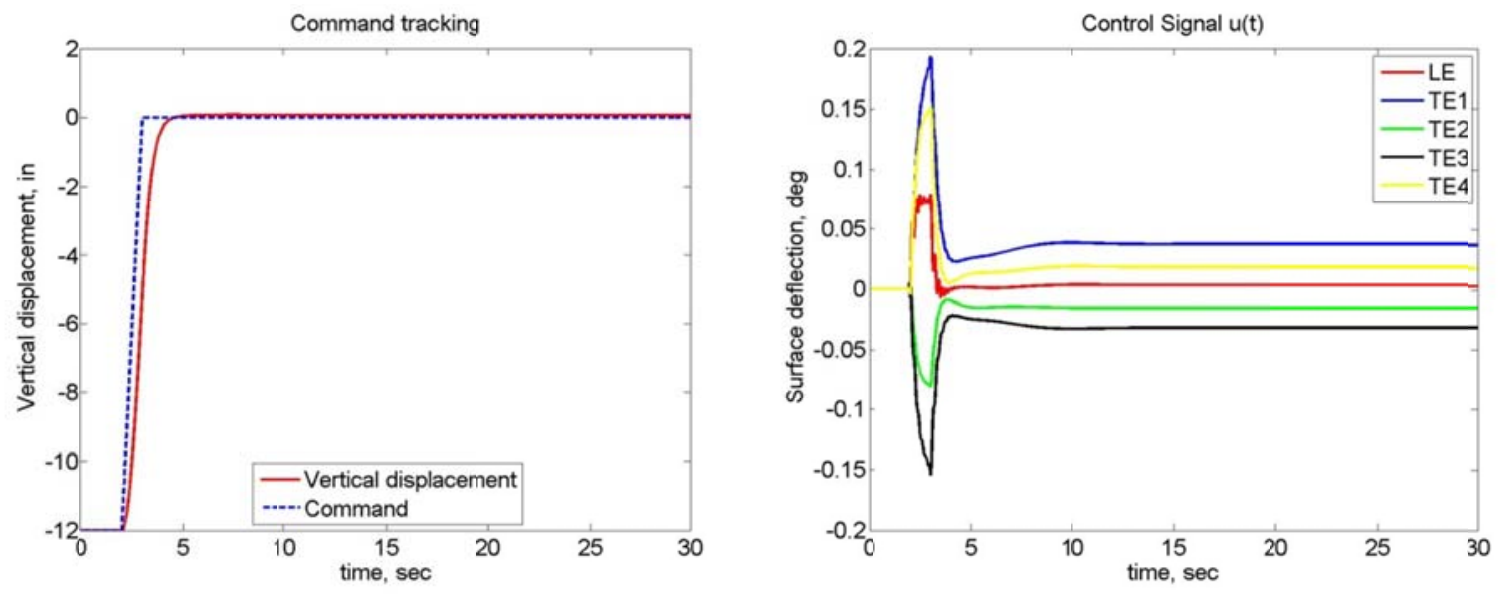
(a) Vertical tunnel position (in) Attitude response

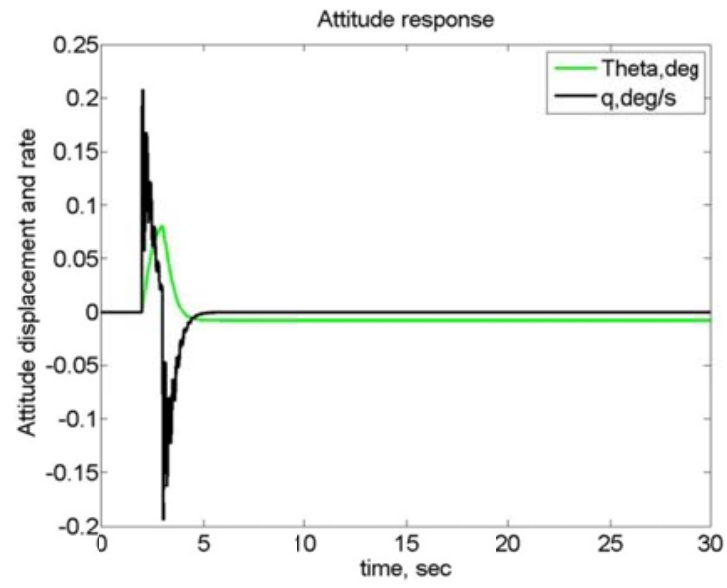

(c) Model attitude $\theta$ (deg) and q (deg/s) (b) Control deflections (deg)

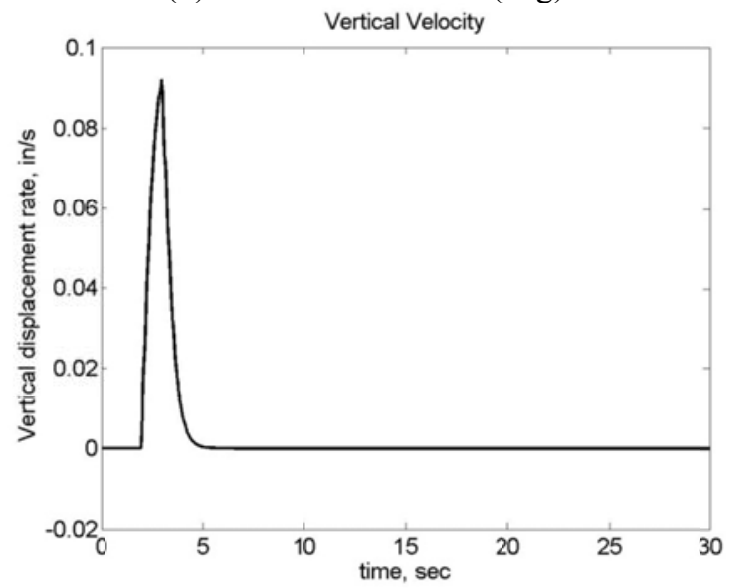

(d) Vertical velocity Vz (in/s)

Figure 5. Measured output responses for the baseline controller (130psf) evaluated at $90 \mathrm{psf}(\mathrm{ir}=\mathbf{8})$.

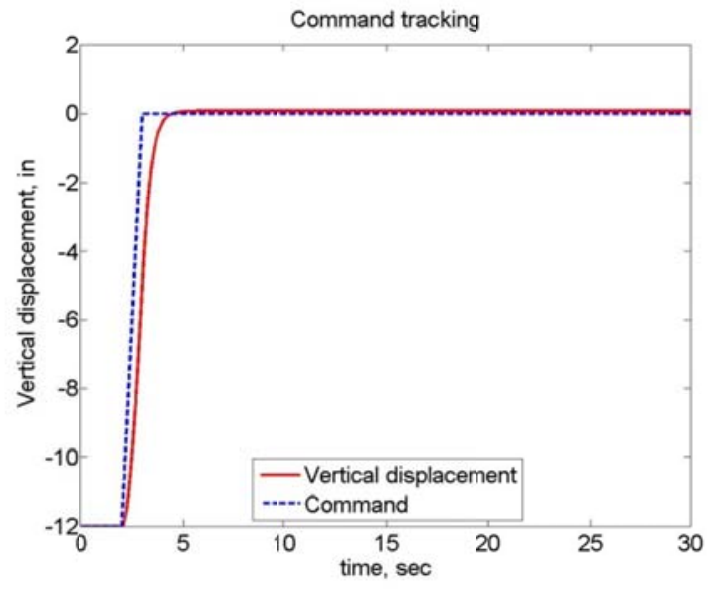

(a) Vertical tunnel position (in)

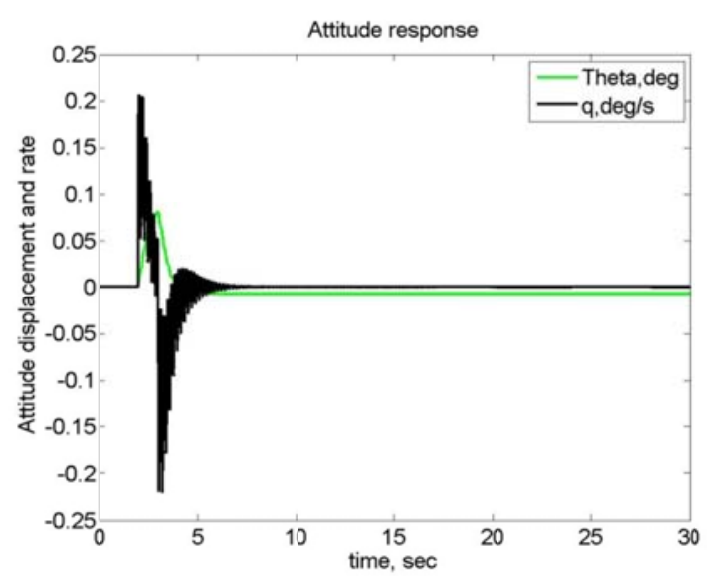

(c) Model attitude $\theta$ (deg) and q (deg/s)

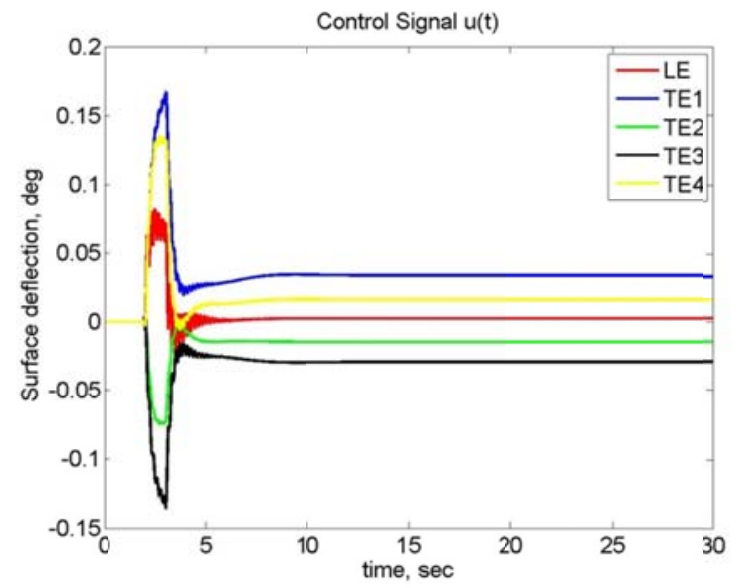

(b) Control deflections (deg)

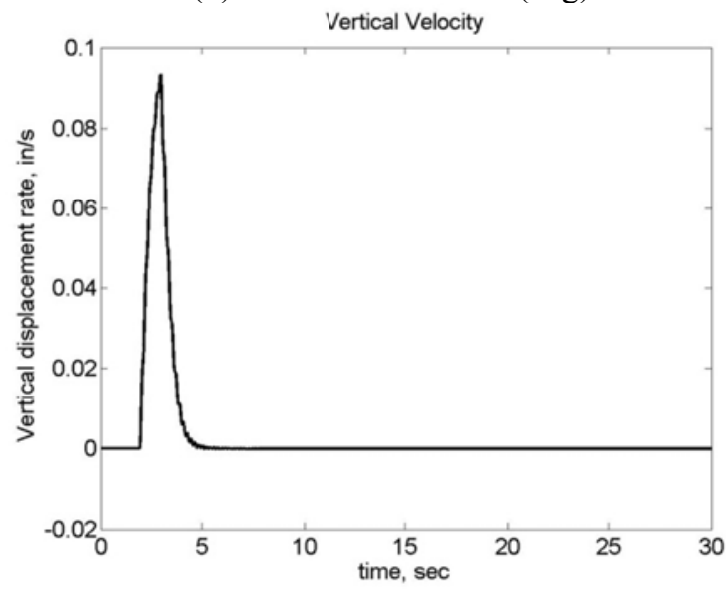

(d) Vertical velocity Vz (in/s)

Figure 6. Measured output responses for the baseline controller (130psf) evaluated at $80 \mathrm{psf}(\mathrm{ir}=9)$. 


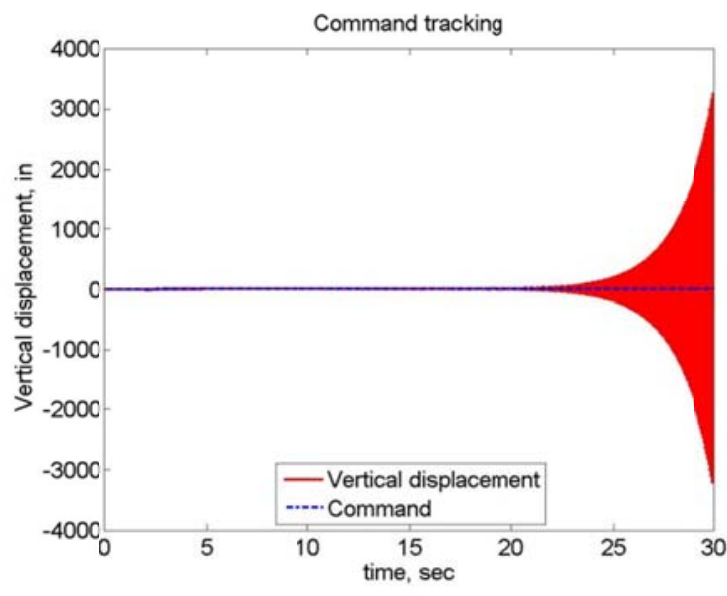

(a) Vertical tunnel position at $70 \mathrm{psf}(\mathrm{ir}=7)$

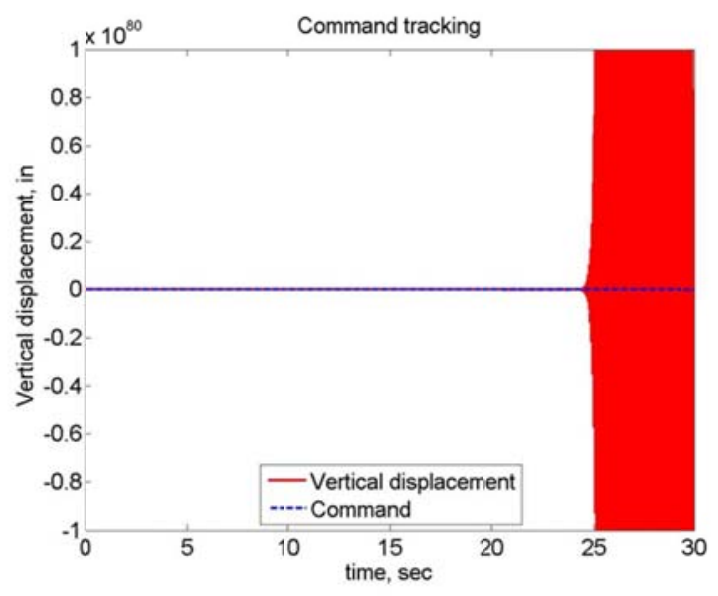

(b) Vertical tunnel position at $60 \mathrm{psf}(\mathrm{ir}=6)$

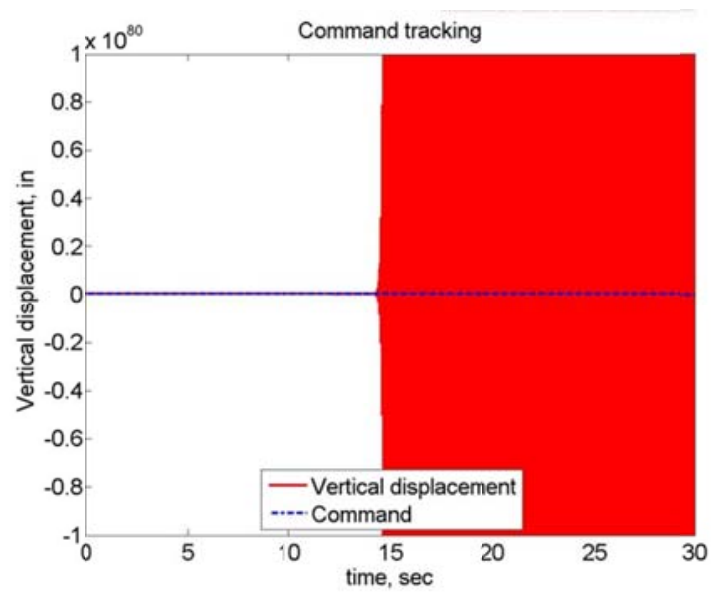

(c) Vertical tunnel position (in) at $50 \mathrm{psf}(\mathrm{ir}=6)$

Figure 7. Measured output responses for the baseline controller (130psf) evaluated at 70, 60, $50 \mathrm{psf}$.

With the $L_{1}$ adaptive augmentation, the augmented controller with parameters $\left(A_{m i}, B_{m i}, C_{m i}\right), i=10(130 \mathrm{psf})$ can stabilize the system when the real full order plant ranges from 130 to $30 \mathrm{psf}$ test conditions, ir changes from 10 to 1 . Figs. 8 to 17 show the system performance of the $L_{1}$ augmented controller dealing with the unknown real plant. Even for the largest change in dynamic pressure from nominal $30 \mathrm{psf}$, which is barely enough to sustain flight, ir $=1$, in Fig. 17 the control signal has small magnitude oscillations. The augmented controller can still stabilize the system and maintain reasonable performance. There is an increase in the control effort as evident from Fig. 8 to Fig. 17 because the $L_{1}$ adaptive estimation can estimate the model mismatch and generate an augmentation control signal to stabilize the system and compensate the effect of the model mismatch in the output. The stability results for the $L_{1}$ adaptive output feedback controller are listed in Table 3 . Simulation results show that a single $L_{1}$ adaptive controller can maintain stability and performance bounds for all 10 test points

The simulation results are encouraging and the methodology is ready to be applied to more challenging nonlinear very flexible vehicle models. 


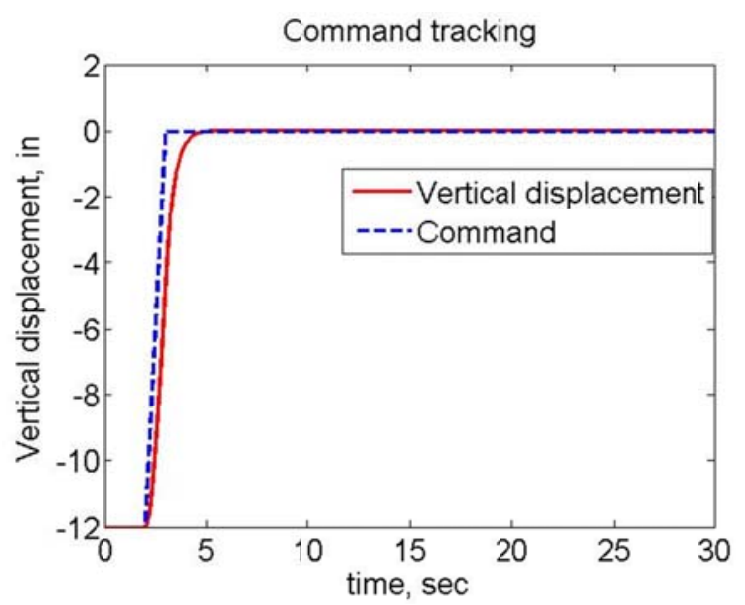

(a) Vertical tunnel position (in)

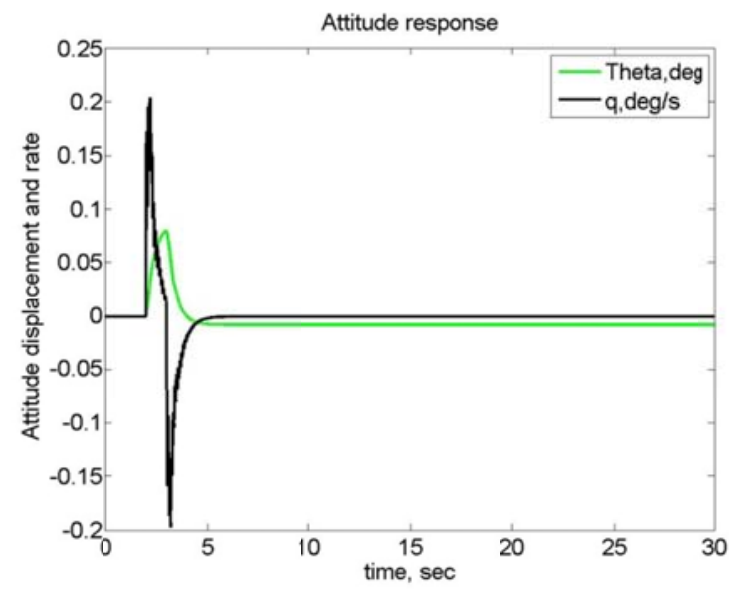

(c) Model attitude $\theta(\mathrm{deg})$ and $\mathrm{q}(\mathrm{deg} / \mathrm{s})$

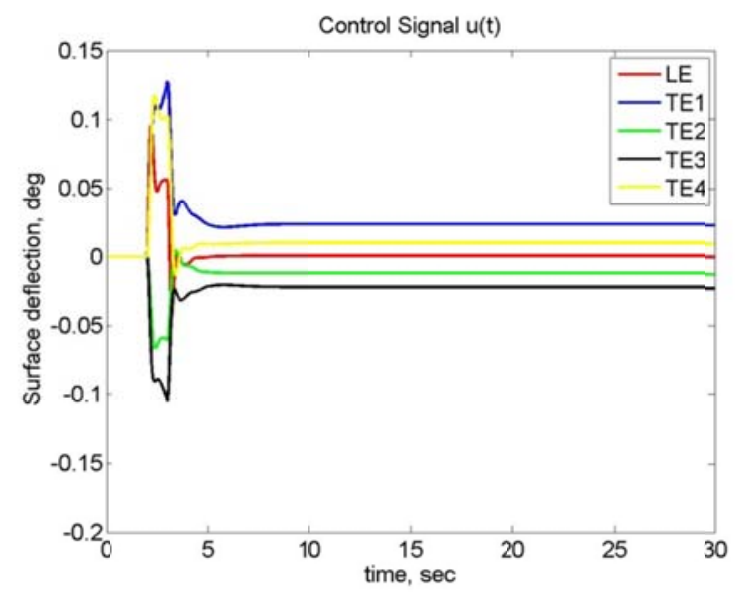

(b) Control deflections (deg)

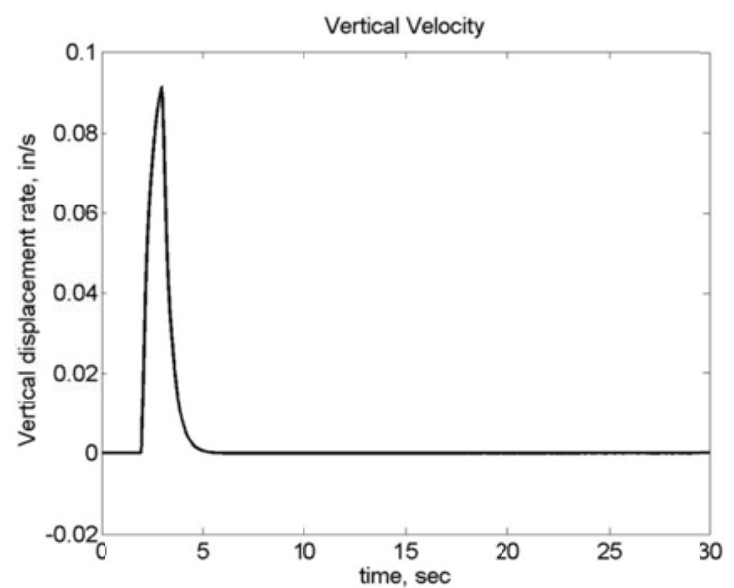

(d) Vertical velocity Vz (in/s)

Figure 8. Measured output responses for the $L_{1}$ adaptive controller (130psf) evaluated at $130 \mathrm{psf}(\mathrm{ir}=10)$.

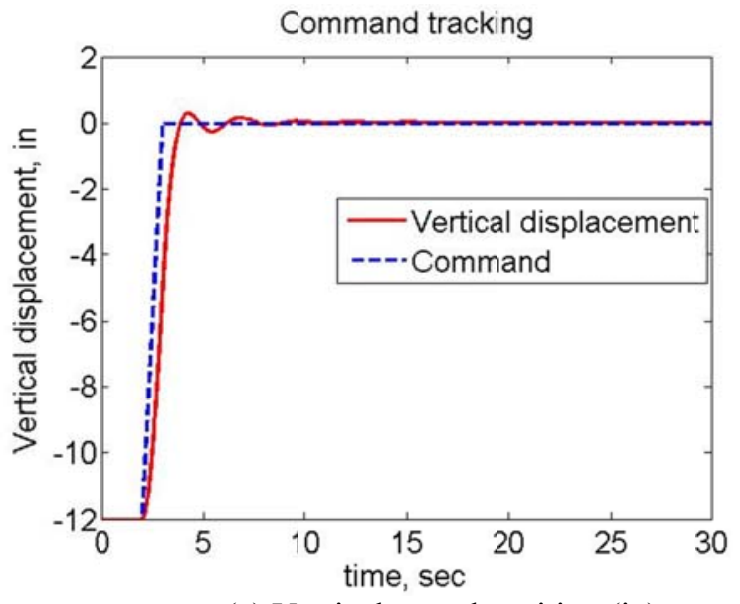

(a) Vertical tunnel position (in)

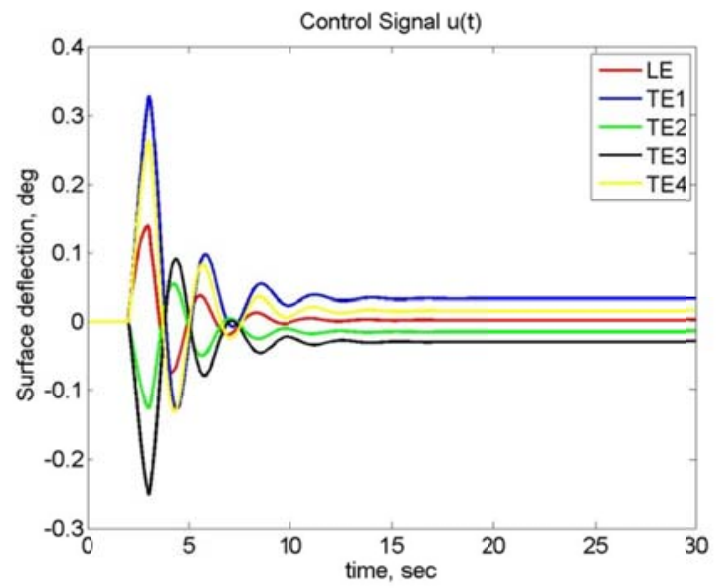

(b) Control deflections (deg) 


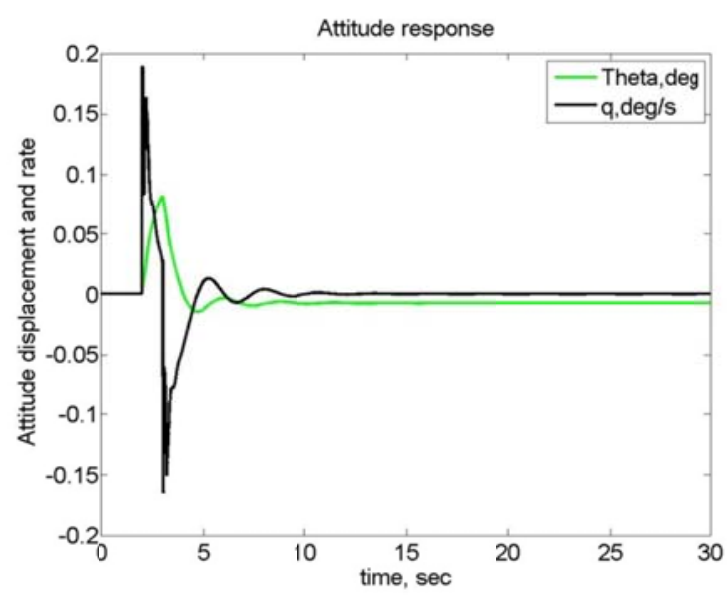

(c) Model attitude $\theta(\mathrm{deg})$ and $\mathrm{q}(\mathrm{deg} / \mathrm{s})$

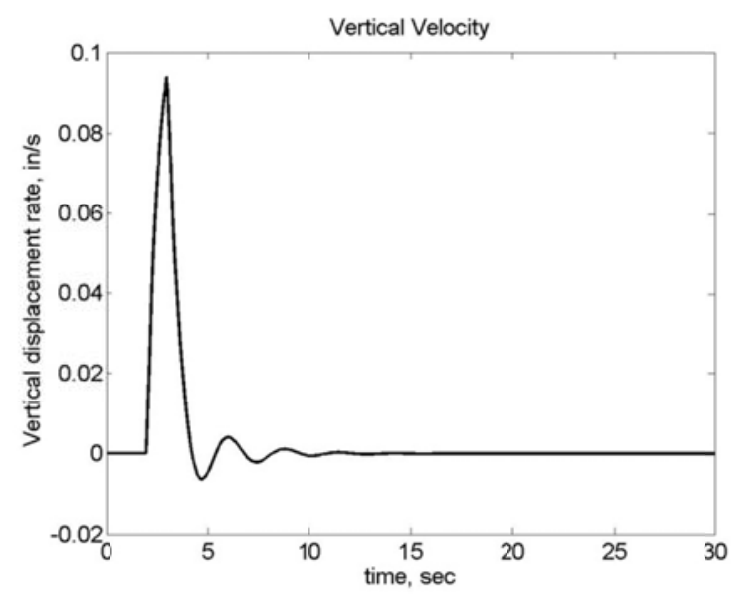

(d) Vertical velocity Vz (in/s)

Figure 9. Measured output responses for the $L_{1}$ adaptive controller (130psf) evaluated at $90 \mathrm{psf}(\mathrm{ir}=9)$.

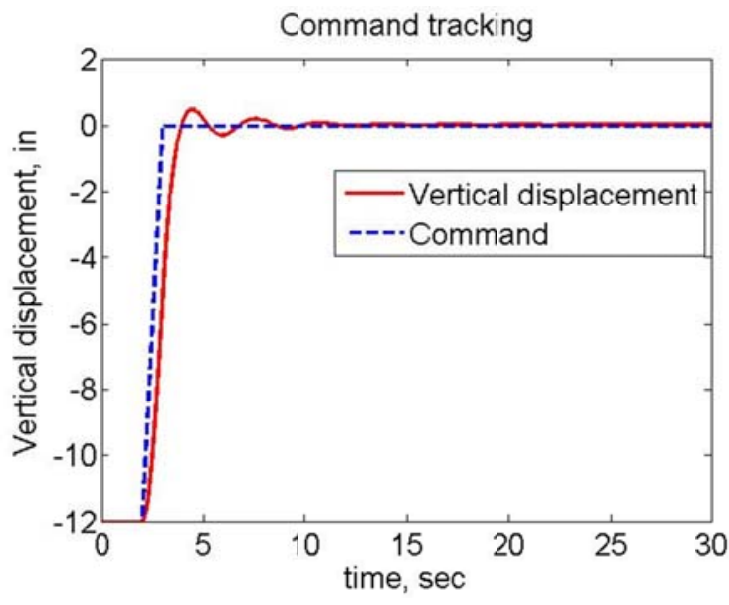

(a) Vertical tunnel position (in)

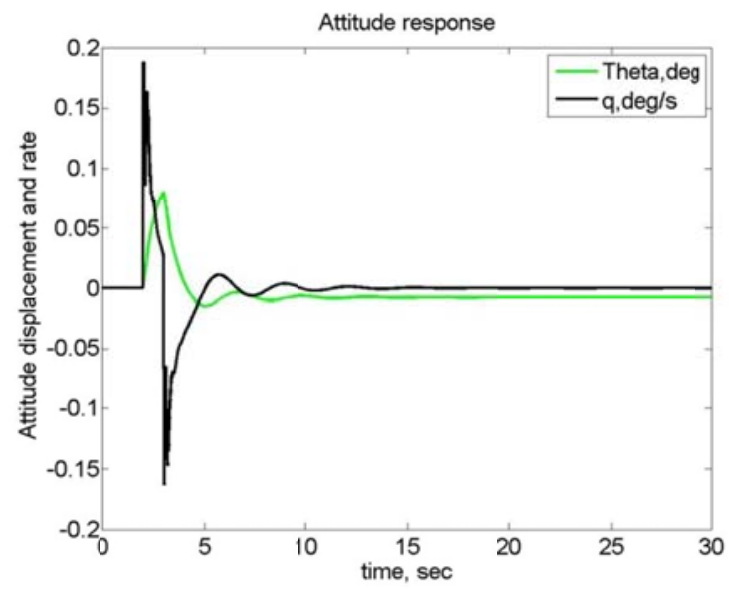

(c) Model attitude $\theta$ (deg) and q (deg/s)

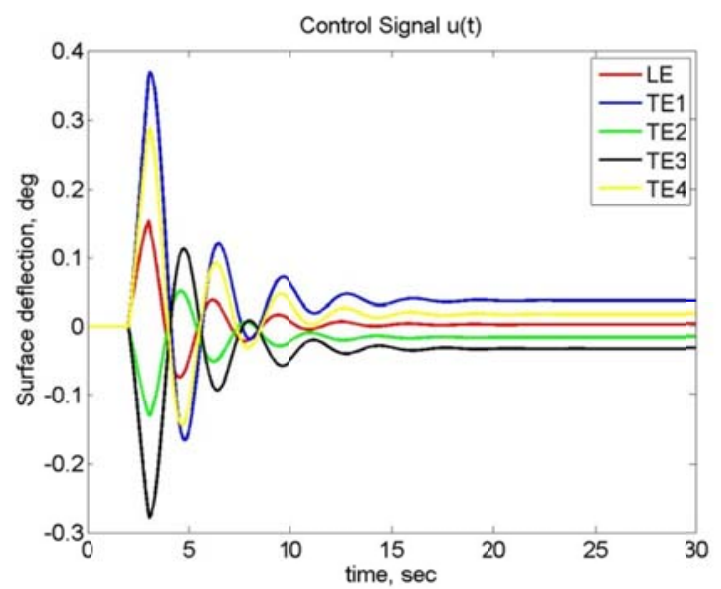

(b) Control deflections (deg)

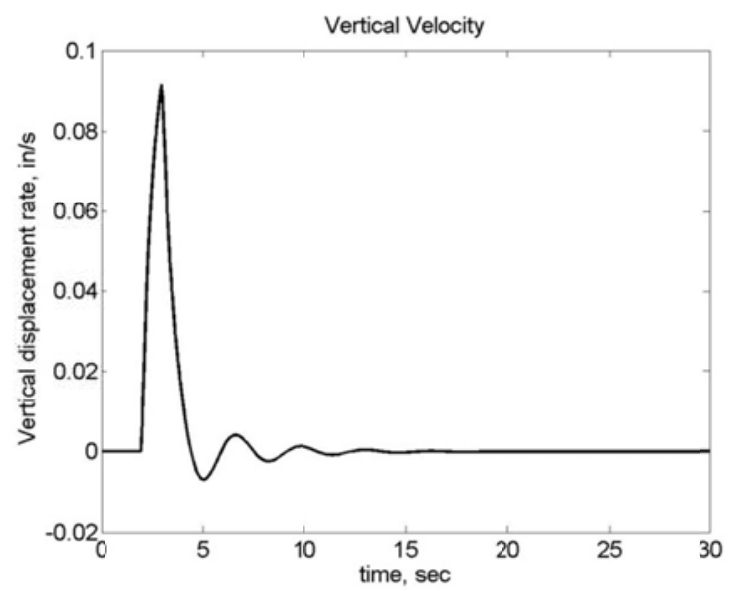

(d) Vertical velocity Vz (in/s)

Figure 10. Measured output responses for the $L_{1}$ adaptive controller (130psf) evaluated at $80 \mathrm{psf}$ (ir $=8$ ). 


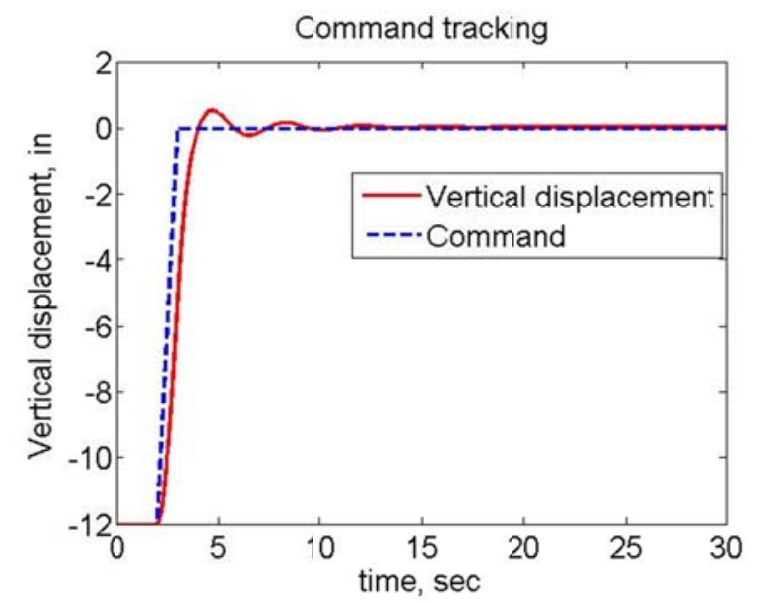

(a) Vertical tunnel position (in)

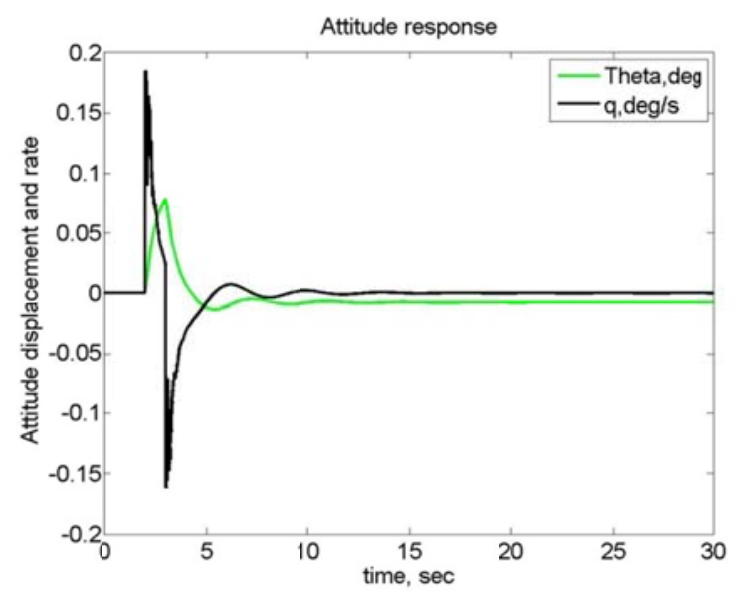

(c) Model attitude $\theta$ (deg) and q (deg/s)

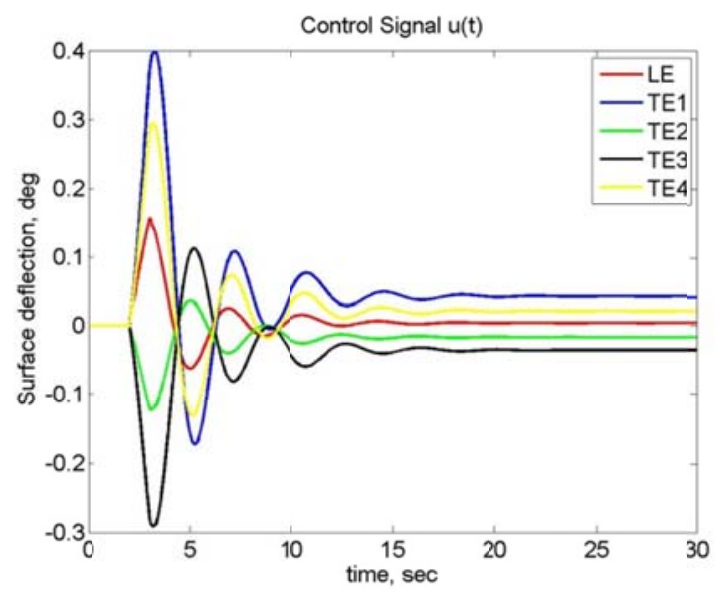

(b) Control deflections (deg)

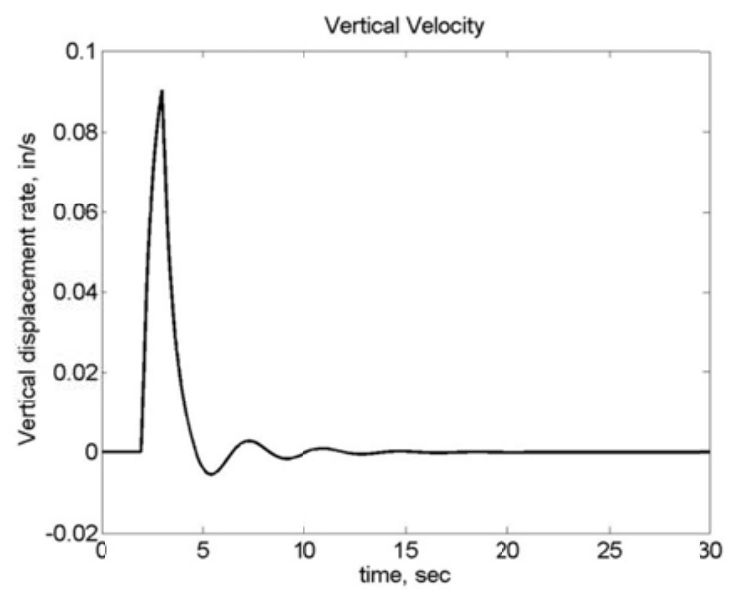

(d) Vertical velocity Vz (in/s)

Figure 11. Measured output responses for the $L_{1}$ adaptive controller (130psf) evaluated at $70 \mathrm{psf}$ (ir $=7$ ).

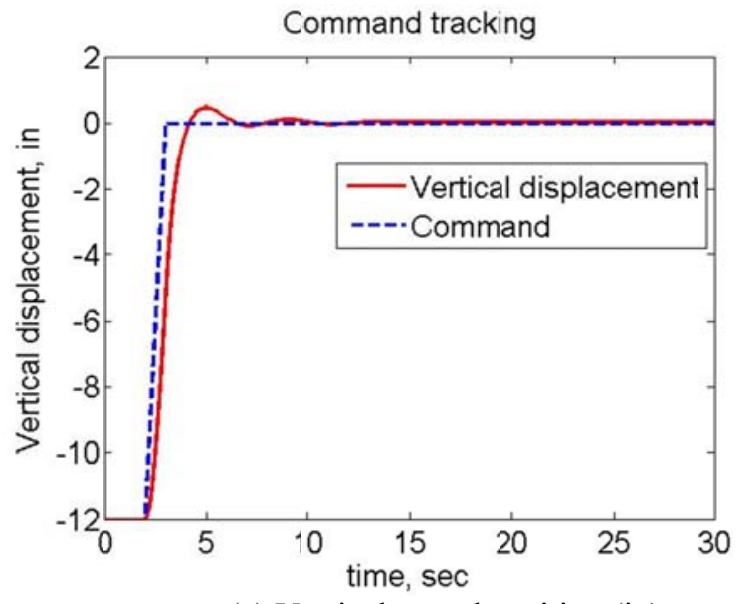

(a) Vertical tunnel position (in)

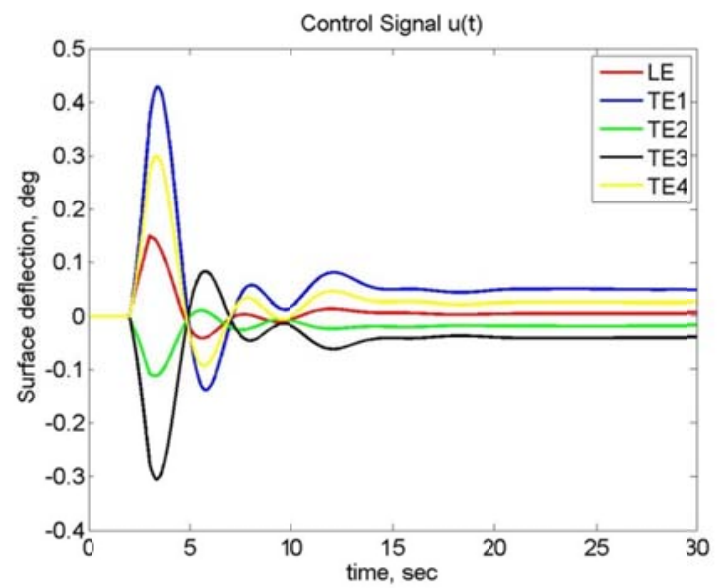

(b) Control deflections (deg) 


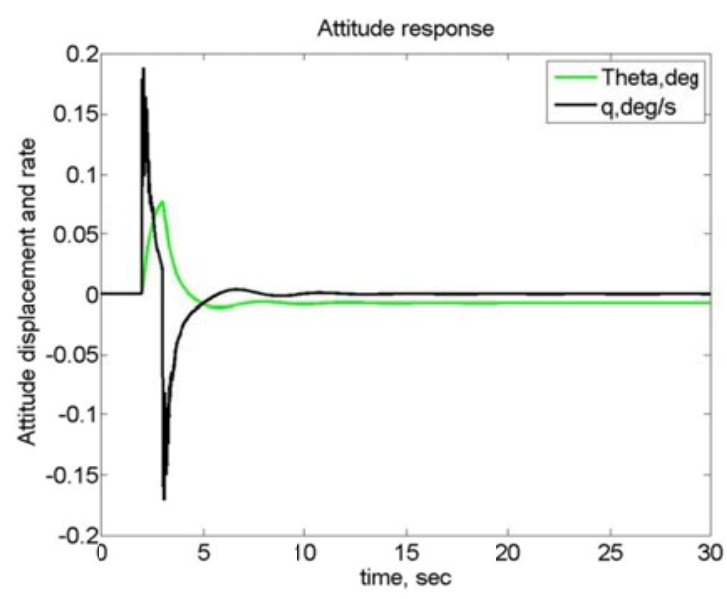

(c) Model attitude $\theta(\mathrm{deg})$ and $\mathrm{q}(\mathrm{deg} / \mathrm{s})$

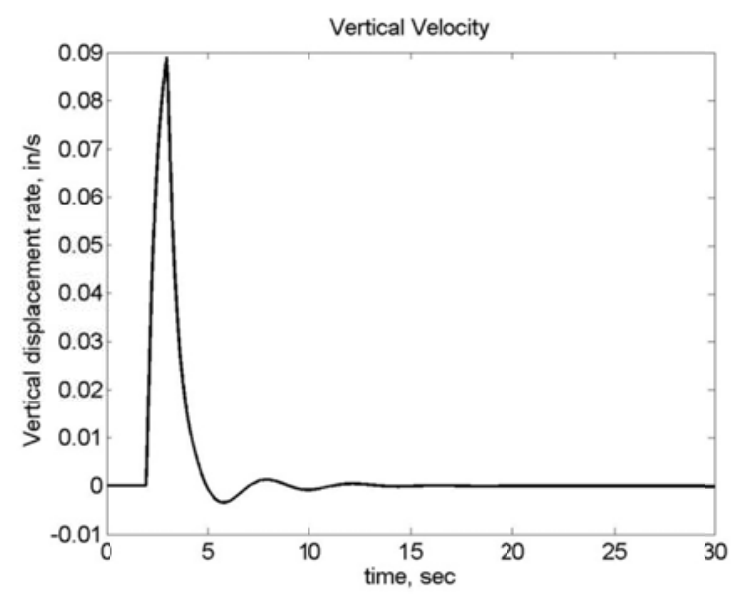

(d) Vertical velocity $\mathrm{Vz}($ in/s)

Figure 12. Measured output responses for the $L_{1}$ adaptive controller (130psf) evaluated at $60 \mathrm{psf}(\mathrm{ir}=6)$.

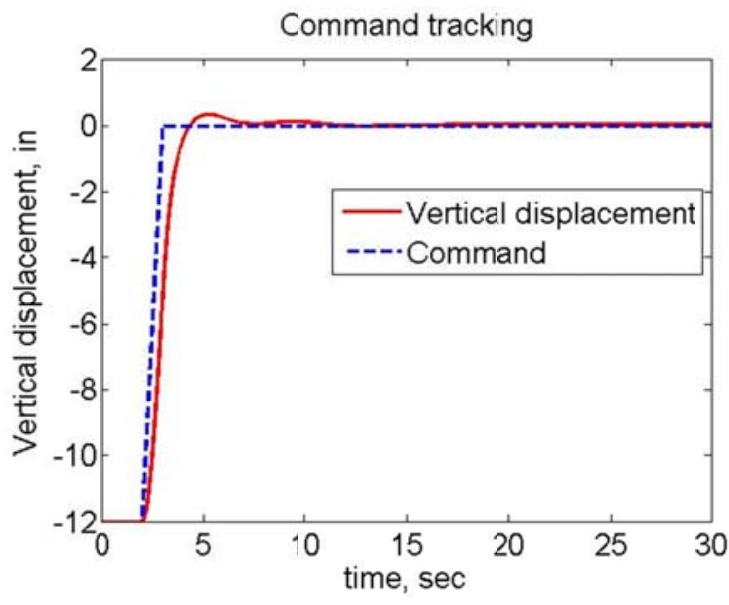

(a) Vertical tunnel position (in)

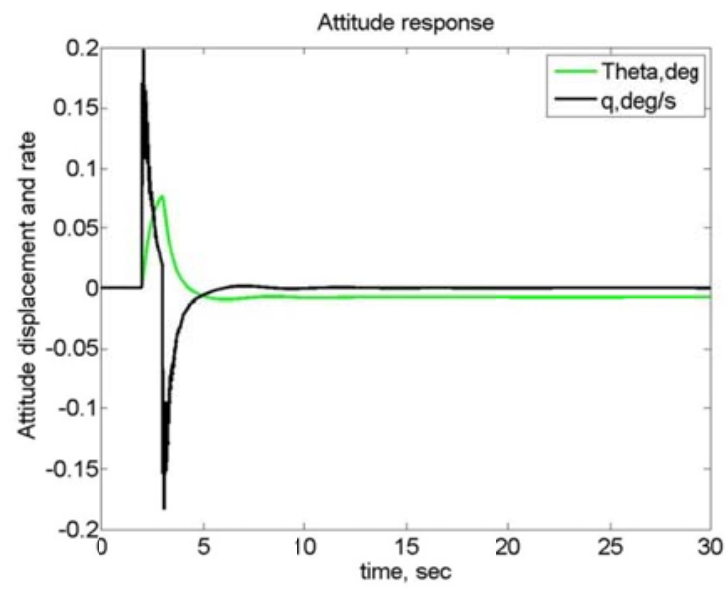

(c) Model attitude $\theta$ (deg) and q (deg/s)

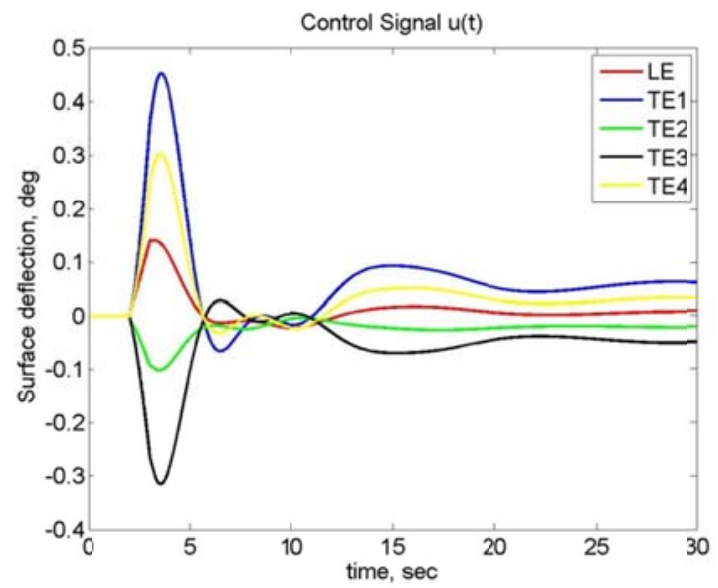

(b) Control deflections (deg)

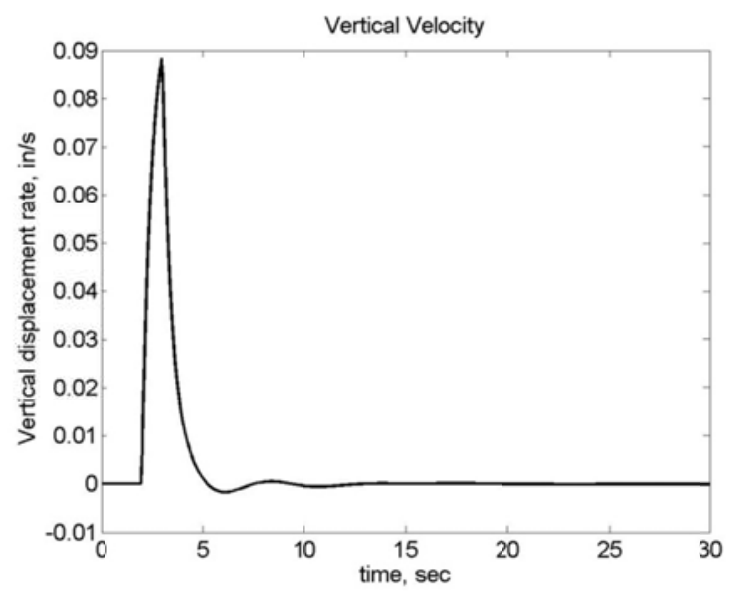

(d) Vertical velocity Vz (in/s)

Figure 13. Measured output responses for the $L_{1}$ adaptive controller (130psf) evaluated at $50 \mathrm{psf}(\mathrm{ir}=5)$. 


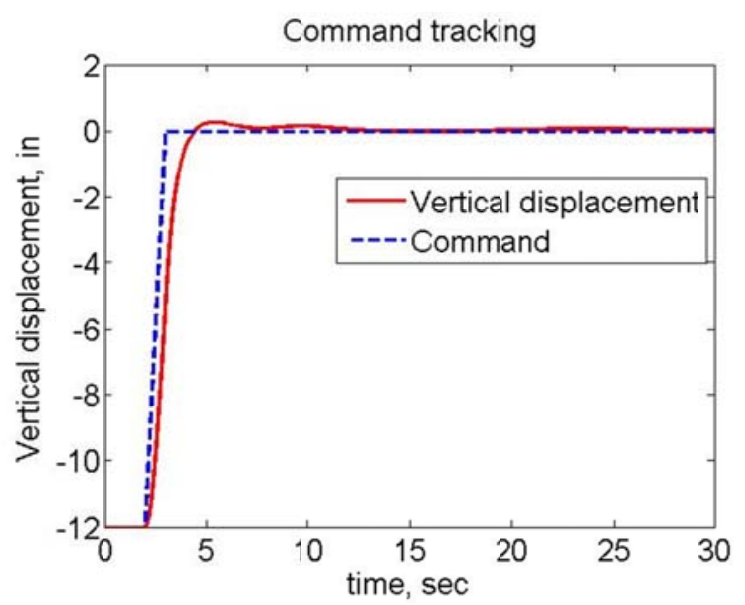

(a) Vertical tunnel position (in)

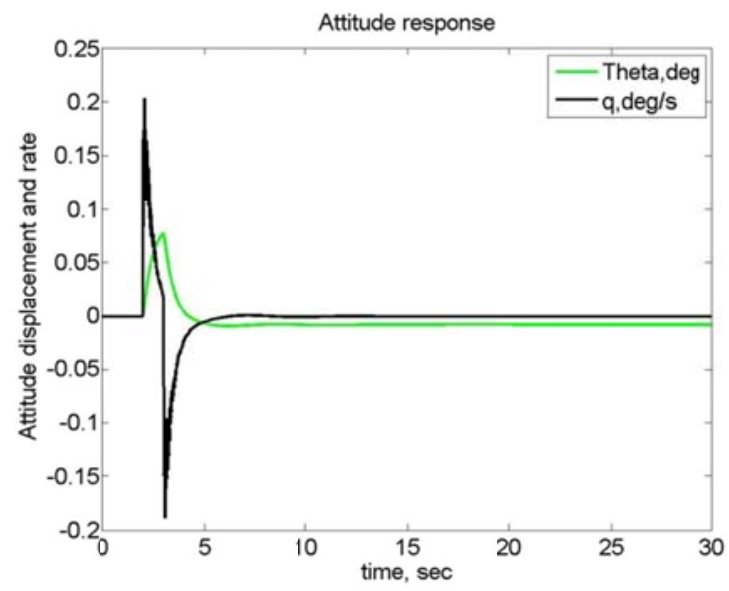

(c) Model attitude $\theta$ (deg) and q (deg/s)

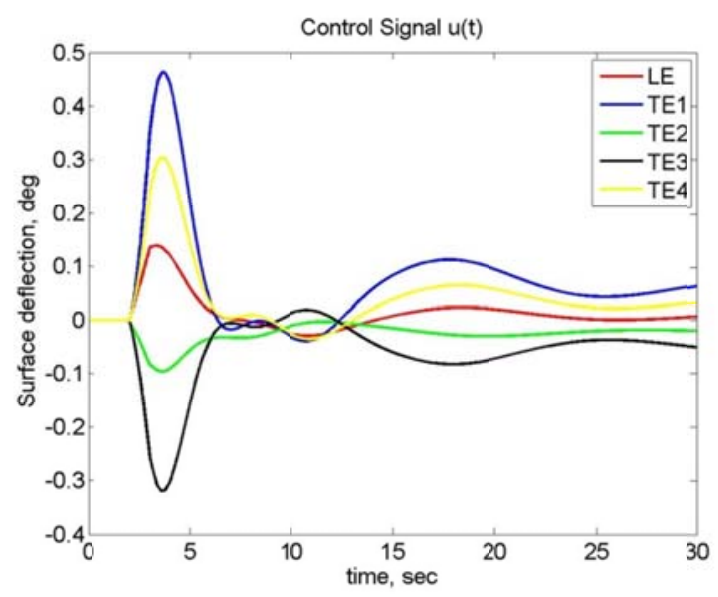

(b) Control deflections (deg)

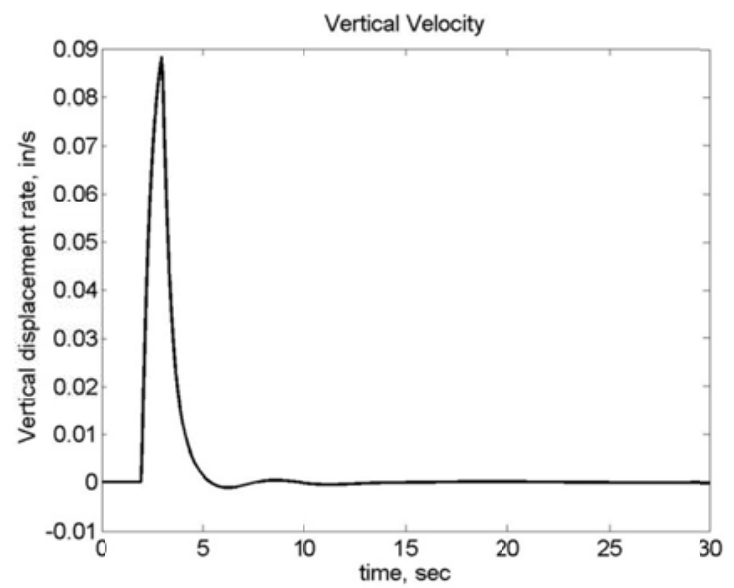

(d) Vertical velocity Vz (in/s)

Figure 14. Measured output responses for the $L_{1}$ adaptive controller (130psf) evaluated at 45 psf (ir $=4$ ).

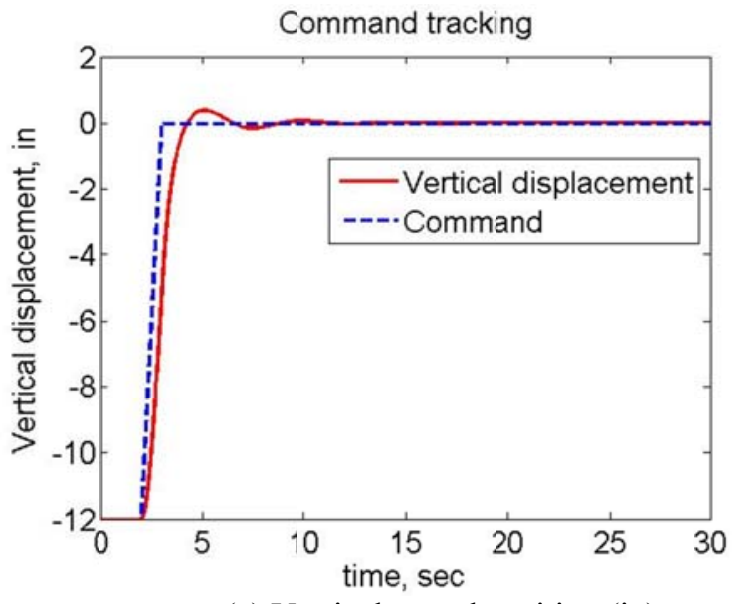

(a) Vertical tunnel position (in)

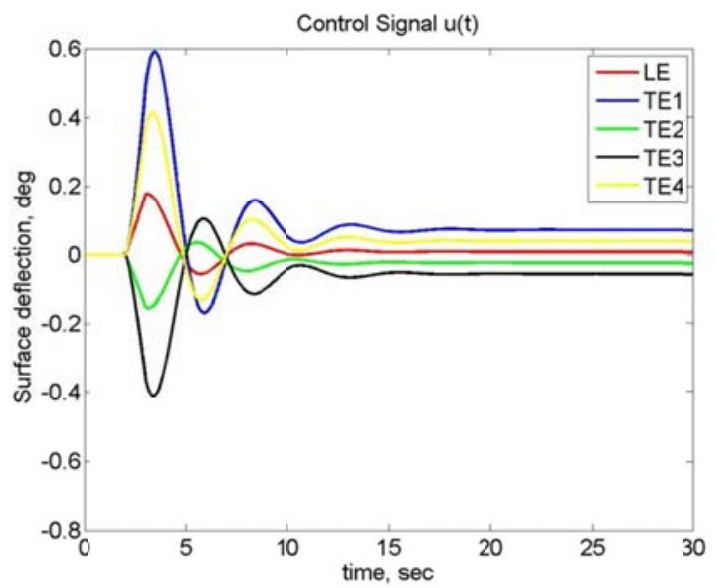

(b) Control deflections (deg) 


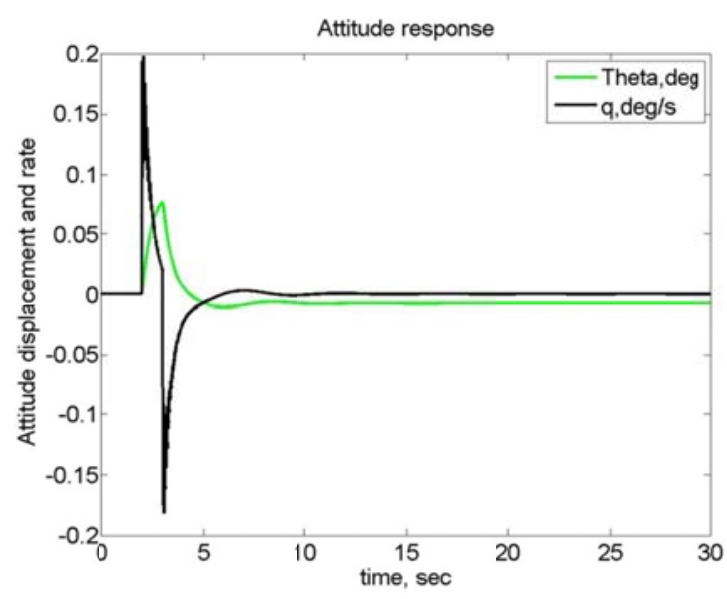

(c) Model attitude $\theta(\mathrm{deg})$ and $\mathrm{q}(\mathrm{deg} / \mathrm{s})$

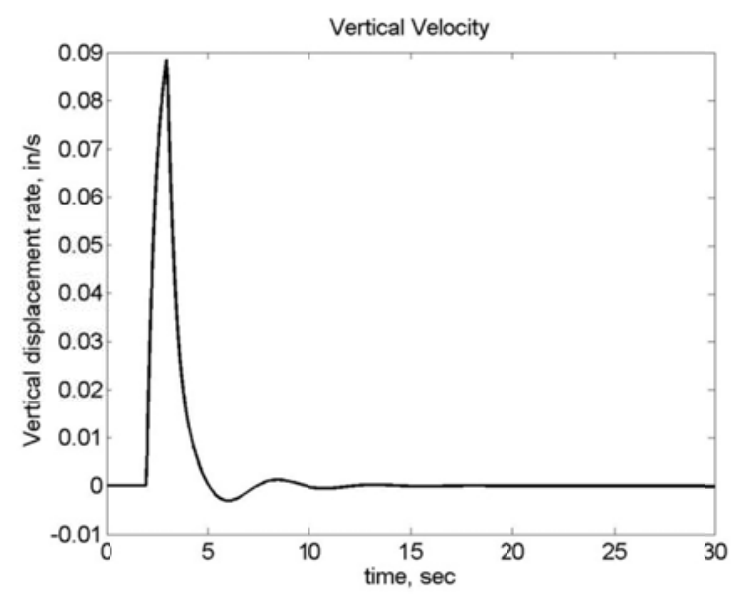

(d) Vertical velocity $\mathrm{Vz}($ in/s)

Figure 15. Measured output responses for the $L_{1}$ adaptive controller (130psf) evaluated at $40 \mathrm{psf}(\mathrm{ir}=3)$.

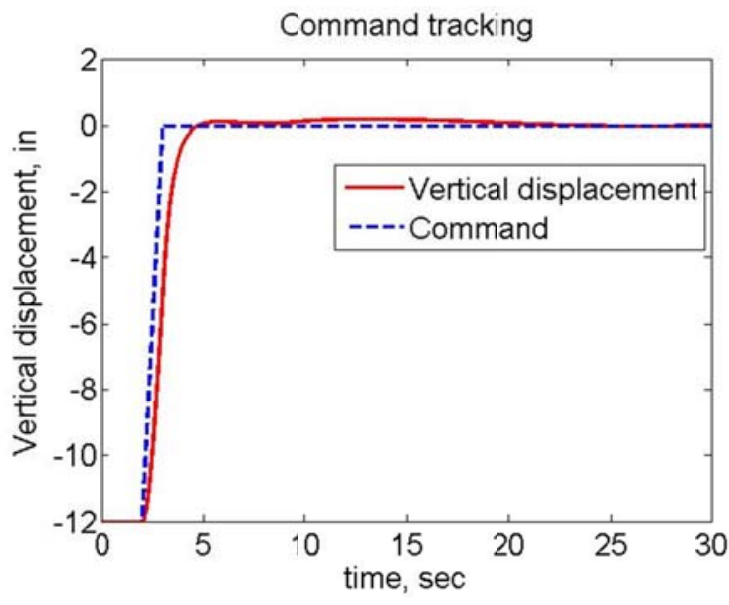

(a) Vertical tunnel position (in)

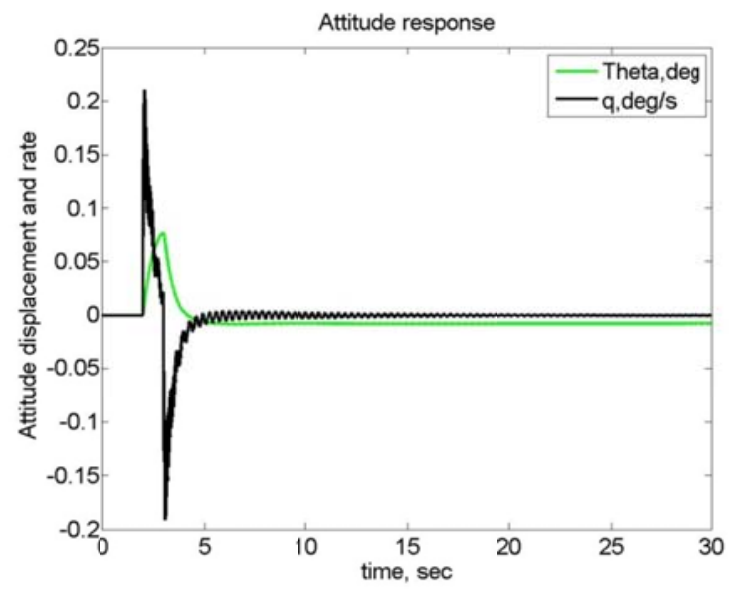

(c) Model attitude $\theta$ (deg) and q (deg/s)

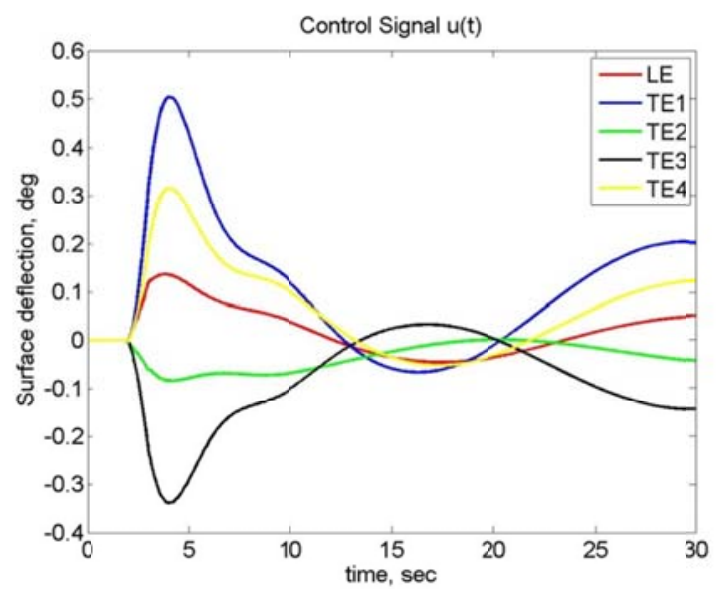

(b) Control deflections (deg)

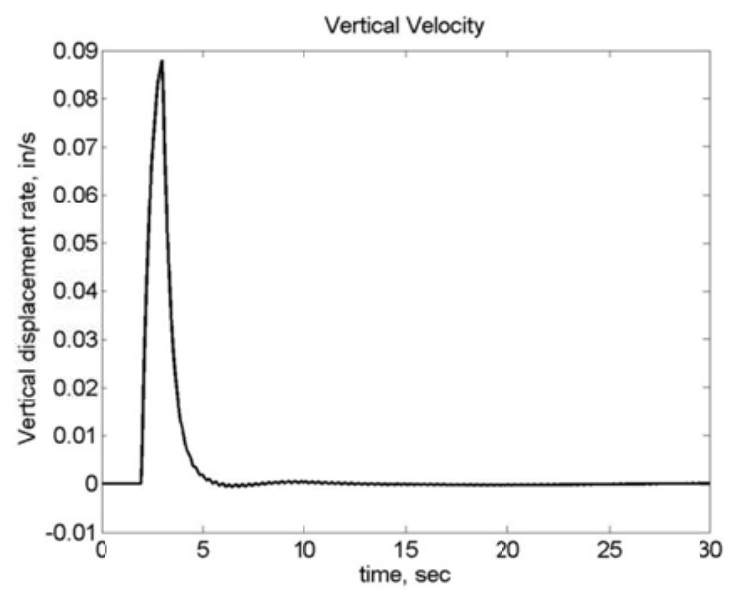

(d) Vertical velocity Vz (in/s)

Figure 16. Measured output responses for the $L_{1}$ adaptive controller (130psf) evaluated at $35 \mathrm{psf}$ (ir $=2$ ). 


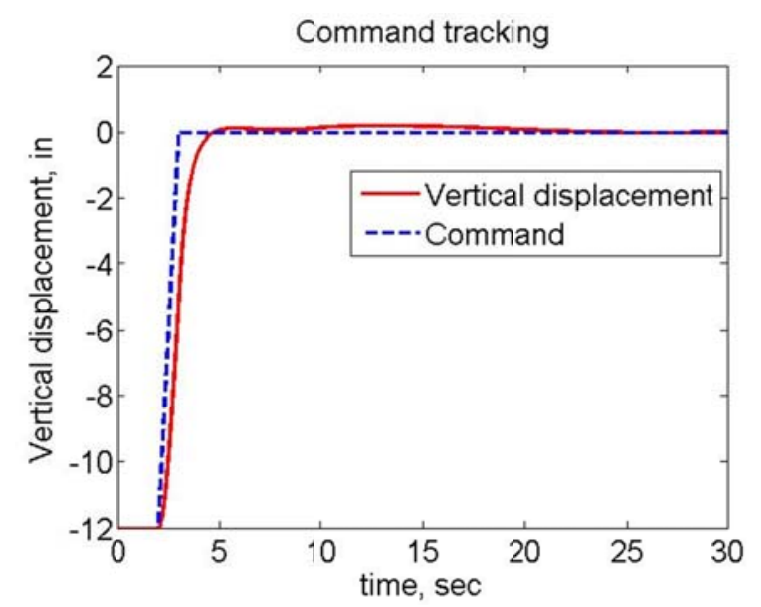

(a) Vertical tunnel position (in)

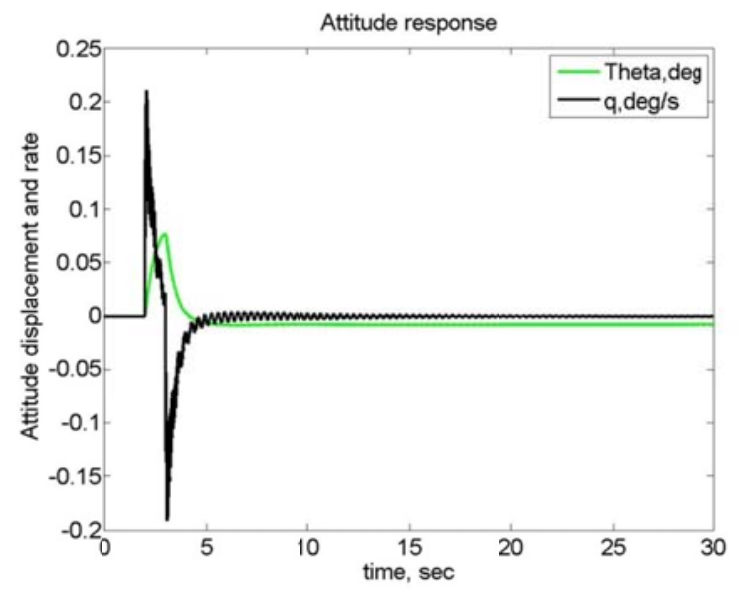

(c) Model attitude $\theta$ (deg) and q (deg/s)

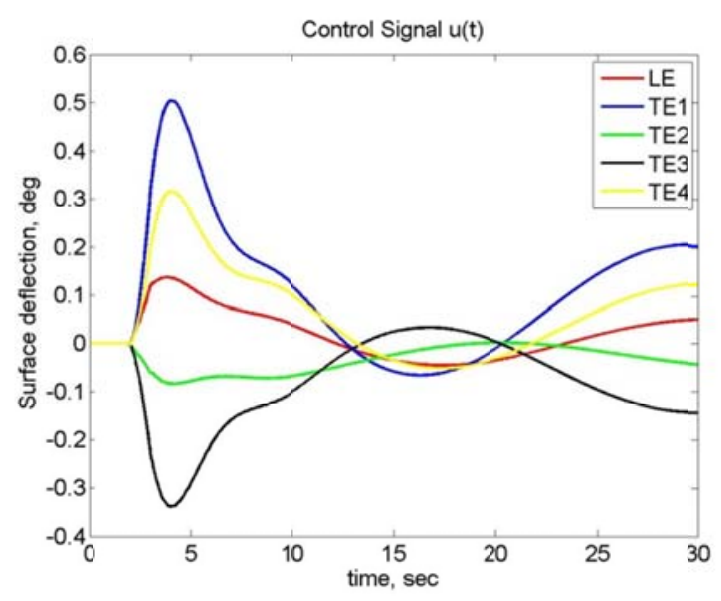

(b) Control deflections (deg)

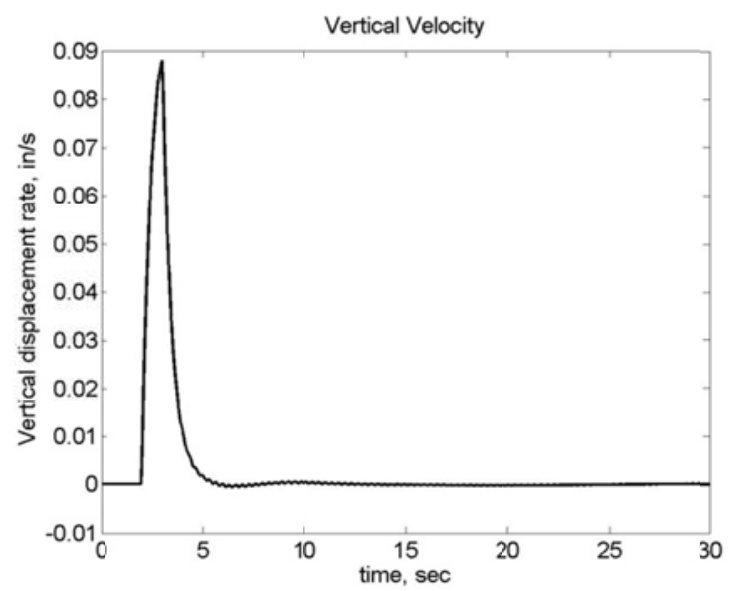

(d) Vertical velocity $\mathrm{Vz}(\mathrm{in} / \mathrm{s})$

Figure 17. Measured output responses for the $L_{1}$ adaptive controller (130psf) evaluated at $30 \mathrm{psf}$ (ir $=1$ ).

Table 3. Stability of $L_{1}$ augmented controller for models at different test conditions.

\begin{tabular}{|c|c|c|c|c|c|c|c|c|c|c|}
\hline \multirow{2}{*}{ Stable /not } & \multicolumn{10}{|c|}{$\mathrm{A}_{\text {ir }}$ from the full state plant } \\
\cline { 2 - 12 } & 1 & 2 & 3 & 4 & 5 & 6 & 7 & 8 & 9 & 10 \\
\hline $\mathrm{Am}=10$ & $\sqrt{ }$ & $\sqrt{ }$ & $\sqrt{ }$ & $\sqrt{ }$ & $\sqrt{ }$ & $\sqrt{ }$ & $\sqrt{ }$ & $\sqrt{ }$ & $\sqrt{ }$ & $\sqrt{ }$ \\
\hline
\end{tabular}

\section{Conclusion}

This paper presents an output feedback $L_{1}$ adaptive control framework applied to an integrated flight/structural mode control of a high aspect ratio, highly flexible, semi-span wind tunnel model capable of pitch and plunge motion. This model exhibits a high level of rigid body flexible mode interactions and full body flutter dynamics. The $L_{1}$ adaptive controller is used as an augmentation on a baseline controller designed at a single test condition. The simulation of the baseline and the $L_{1}$ adaptive control conglomeration show promising results. The addition of an $L_{1}$ adaptive controller stabilizes the model when the baseline alone cannot do the job. The augmented controller designed at a single test condition can maintain stability for a wide range of dynamic pressures, all 10 of the test conditions. 
While the results are preliminary since the evaluation models are linear with no significant time delay in the system, they are nevertheless encouraging and warrant further application to more sophisticated fully nonlinear very flexible aircraft models.

\section{Acknowledgments}

The authors would like to thank John Cooper, Jie Luo in AIM lab at University of Connecticut for their comments and suggestions.

\section{References:}

[1] Del Rosario, R, G. Follen, R. Wahls, "NASA Subsonic Fixed Wing Project Annual Review," Fundamental Aeronautics Program Annual Review, 18-19 November 2010.

[2] Kharisov, E., N. Hovakimyan, and K. Astrom, Comparison of Several Adaptive Controllers According to Their Robustness Metrics, In Proceedings of AIAA Guidance, Navigation and Control Conference, Toronto, Canada, AIAA-2010$8047,2010$.

[3] Cao, C. and N. Hovakimyan, "Design and Analysis of a Novel L1 Adaptive Control Architecture with Guaranteed Transient Performance," IEEE Transactions on Automatic Control, vol.53, No.2, pp. 586-591, 2008.

[4] Cao, C. and N. Hovakimyan, "L1 Adaptive Controller for Systems with Unknown Time-varying Parameters and Disturbances in the Presence of Non-zero Trajectory Initialization Error", International Journal of Control, Vol. 81, No. 7, 11471161, July 2008.

[5] Cao, C. and N. Hovakimyan, "Stability Margins of L1 Adaptive Controller," IEEE Transactions on Automatic Control, vol. 55, No. 2, pp. 480-487, 2010.

[6] Cao, C. and N. Hovakimyan, "L1 Adaptive Controller for a Class of Systems with Unknown Nonlinearities: Part I," American Control Conference, Seattle, WA, pp. 4093-4098, 2008.

[7] Cao, C. and N. Hovakimyan, "L1 Adaptive Controller for Nonlinear Systems in the Presence of Unmodeled Dynamics: Part II," American Control Conference, Seattle, WA, pp. 4099-4104, 2008.

[8] Gregory, I. M. "From Dynamically Scaled High Risk Flight Testing to Learn-to-Fly: Control Enabled Capabilities for Autonomous Flight,", Aerospace Decision and Control Workshop, Georgia Institute of Technology, 11-12 June 2012.

[9] Cao, C., Naira Hovakimyan, "L1 Adaptive Output-Feedback Controllers for Non-Strictly-Positive-Real Reference Systems: Missile Longitudinal Autopilot Design.” Journal of Guidance, Control, and Dynamics, vol. 32, No. 3, pp.717-726, MayJune, 2009.

[10] Gregory, I.M., C. Cao, V. Patel and N. Hovakimyan, "Adaptive Control Laws for Flexible Semi-Span Wind Tunnel Model of High-Aspect Ratio Flying Wing," in Proceedings of AIAA Guidance, Navigation and Control Conference, AIAA2007-6525.

[11] Gregory, I. and Xargay, E. and Cao, C. and Hovakimyan, N. "Flight Test of L1 Adaptive Controller on the NASA AirSTAR Flight Test Vehicle," in Proceedings AIAA Guidance, Navigation and Control Conference 2010.

[12] Hovakimyan, N. and Cao, C. L1 Adaptive Control Theory: Guaranteed Robustness with Fast Adaptation. Siam 2010. 